\title{
NK cell survival mediated through the regulatory synapse with human DCs requires IL-15R $\alpha$
}

\author{
Fabienne Brilot, Till Strowig, Susanne M. Roberts, Frida Arrey, and Christian Münz
}

Laboratory of Viral Immunobiology and Christopher H. Browne Center for Immunology and Immune Diseases, Rockefeller University, New York, New York, USA.

\begin{abstract}
DCs activate NK cells during innate immune responses to viral infections. However, the composition and kinetics of the immunological synapse mediating this interaction are largely unknown. Here, we report the rapid formation of an immunological synapse between human resting NK cells and mature DCs. Although inhibitory NK cell receptors were polarized to this synapse, where they are known to protect mature DCs from NK cell lysis, the NK cell also received activation signals that induced mobilization of intracellular calcium and CD69 upregulation. The high-affinity component of the receptor for IL-15, IL-15R $\alpha$, accumulated at the synapse center on NK cells, and blocking of IL-15R $\alpha$ increased NK cell apoptosis and diminished NK cell survival during their interaction with DCs. Furthermore, IL-15R $\alpha$-deficient NK cells, obtained from donors with a history of infectious mononucleosis, showed diminished survival in culture with DCs. Synapse formation was required for IL-15R $\alpha$-mediated NK cell survival, because synapse disruption by adhesion molecule blocking decreased DC-induced NK cell survival. These results identify what we believe to be a novel regulatory NK cell synapse with hallmarks of spatially separated inhibitory and activating interactions at its center. We suggest that this synapse formation enables optimal NK cell activation by DCs during innate immune responses.
\end{abstract}

\section{Introduction}

NK cells are lymphocytes, which have originally been characterized by their ability to kill target cells (1). Activation of target cell recognition by NK cells is determined by the balance between inhibitory and activating signaling pathways (2). In humans, NK cells express several activating receptors, such as NKG2D and natural cytotoxicity receptors, capable of recognizing target cells via cellular stress-, infection-, or transformation-induced molecules on the cell surface (2). On the other hand, most NK cell inhibitory receptors recognize $\mathrm{MHC}$ class I molecules, which are expressed by almost all nucleated cells. The binding of MHC class I complexes to killer cell Ig-like receptors (KIRs) or to the C-lectin-type heterodimeric CD94/NKG2A receptor initiates inhibitory pathways that can override activation signals $(3,4)$. Thus, conjugate formation between NK cells and target cells will lead to cytolytic or noncytolytic interactions. During cell-to-cell contact, supramolecular activating clusters (SMACs) or supramolecular inhibitory clusters (SMICs) accumulate at the contacting plasma membrane between NK cells and target cells within the so-called NK cell immunological synapse (NKIS) (5). During NKIS formation, many proteins, such as adhesion molecules, localize to the cell-to-cell contact area (6). Early translocations of talin and leukocyte function-associated antigen-1 (LFA-1) to the synapse and then to the periphery of the SMAC or SMIC have been observed in cytolytic and noncytolytic conjugates (6-9). However, other molecules or complexes such as perforin and F-actin segregate differently in cytolytic and noncytolytic interac-

Nonstandard abbreviations used: DIC, differential interference contrast; IL-15R, IL-15 receptor; IM, infectious mononucleosis; imDC, immature DC; KIR, killer cell Ig-like receptor; LFA, leukocyte function-associated antigen; $\mathrm{mDC}$, mature DC; NKIS, NK cell immunological synapse; poly(I:C), polyinosine-polycytidylic acid; SMAC, supramolecular activating cluster; SMIC, supramolecular inhibitory cluster. Conflict of interest: The authors have declared that no conflict of interest exists. Citation for this article: J. Clin. Invest. 117:3316-3329 (2007). doi:10.1172/JCI31751. tions $(8,10-12)$. Therefore, the outcome of the interaction between NK and target cells is determined by the NKIS content.

NK cells do not solely mediate cytotoxicity. In humans, 2 main subsets, differing in their functions, have been characterized. $\mathrm{CD} 56^{\text {dim }} \mathrm{CD} 16^{+} \mathrm{NK}$ cells readily lyse targets cells, but secrete low level of cytokines. In contrast, CD56 $6^{\text {bright }} \mathrm{CD} 16^{-} \mathrm{NK}$ cells produce large amounts of cytokines upon stimulation, but acquire cytotoxicity only after prolonged activation (13-15). Interestingly, mature DCs (mDCs) primarily activate the latter NK cell subset $(16,17)$, but the composition of the DC/NK cell synapse in comparison to SMACs and SMICs has not been analyzed so far.

Meanwhile, this DC/NK cell interaction is essential, because it has become increasingly clear that NK cells require activation by DCs to reach their full functional potential (18-20). Previous reports have shown that resting NK cells produce cytokines, proliferate, and increase their cytotoxicity upon activation by both myeloid and plasmacytoid DCs (21). The resulting NK cell activation by DCs is thought to limit pathogen invasion until the adaptive immune system establishes long-lasting immune control. In vivo, DCs and NK cells are colocalized and capable of interacting at the sites of inflammation and in lymph nodes $(16,22,23)$, and it has been shown that the interaction between these 2 cell types is improved when $\mathrm{mDCs}$ and $\mathrm{NK}$ cells have direct cell-to-cell contact (24-26). Although still controversial, several factors have been suggested as essential for the activation of NK cells by DCs. Involvement of soluble or membrane-presented cytokines such as IFN- $\alpha$, IL-12, IL-18, and IL-15 have recently been reported, the latter more specifically associated with NK cell proliferation and survival (24, 27). Biological effects of IL-15 are achieved mainly by its binding to the trimeric IL-15 receptor (IL-15R) complex (IL-15R $\alpha \beta \gamma_{c} ; \mathrm{Kd}$, $\left.10^{-11} \mathrm{M}\right)$. The IL-15R $\beta$ and $\gamma_{c}$ chains are shared with IL-2R, but the IL-15R $\alpha$ chain is a private binding molecule specific for the highaffinity trimeric IL-15R. Consistent with the role of IL-15 in NK 


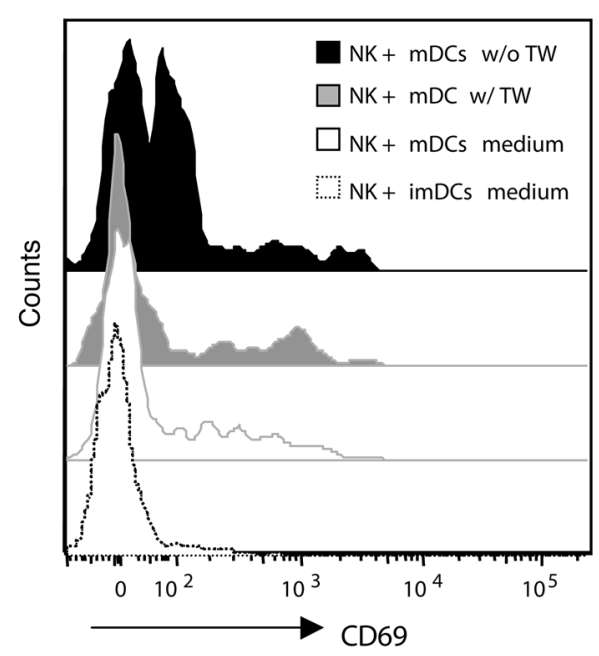

cell physiology, IL-15-/- and IL-15R $\alpha^{-/-}$mice lack NK cells $(28,29)$. Although IL-15R $\alpha$ has a wide tissue expression, its role and localization in immune cell interactions is still unclear (30-32).

Conjugates between $\mathrm{mDCs}$ and resting NK cells are thus essential for the activation of NK cell responses, because the contact with MHC class I ${ }^{\text {high }} \mathrm{mDC}$ does not lead to inhibition of NK cells but to their activation to secrete cytokines, to proliferate, and to

\section{Figure 1}

Cell-to-cell contact of NK cells and mDCs induced CD69 upregulation. Purified blood NK cells were cocultured for $24 \mathrm{~h}$ together with poly(I:C)matured DCs or separated by transwell (TW) membranes preventing cell-to-cell contacts. NK cells cultured alone in media from $\mathrm{mDC}$ or imDC cultures were used as negative controls. Cells were then stained with anti-CD69 and analyzed by flow cytometry. NK cells were gated on size, 7AAD-, CD3- ${ }^{-}, \mathrm{CD}^{-} 6^{+}$, and CD56 bright. Shown is 1 representative experiment of 3 .

become more cytotoxic. Although polarization of IL-12 (33), IL-18 (34), and adhesion molecules (33) in NK cells conjugated with DCs has been previously described in 2-dimensional analysis, the spatial organization and kinetics of the NKIS remain undefined, but have to provide the physical environment during interactions between MHC class I high cells such as mDCs and killer cells such as NK cells to result in activating signals, which do not trigger cytolysis.

In this study, we describe that the optimal activation of NK cells by $\mathrm{mDCs}$ required the establishment of a functional synapse and the rapid organization of a regulatory NKIS between these 2 cell types. The NKIS with DCs displayed phenotypical features of SMICs but transmitted activating signals for NK cell stimulation, with polarized inhibitory NK cell receptors and IL-15R $\alpha$ in separate domains at the central supramolecular cluster of NK cells. The accumulation of IL-15R $\alpha$ at the synapse assured NK cell survival and apoptosis escape after interactions with mDCs. Our
A

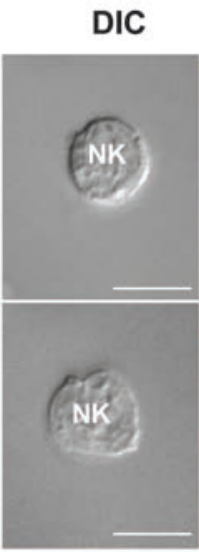

C

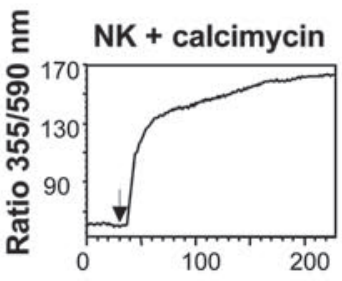

NK cells

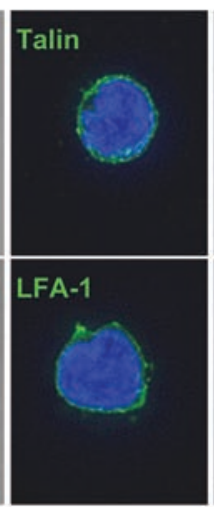

overlay

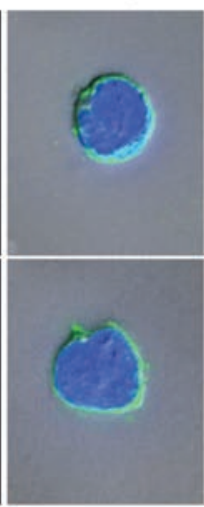

B

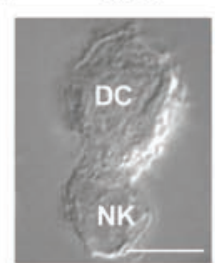

$\mathrm{NK}+\mathrm{DC}$
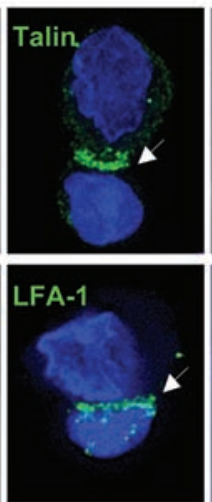

overlay
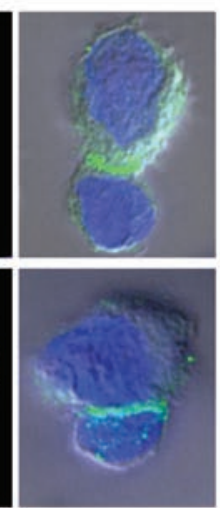

$6 \mathrm{~min}$

$1 \mathrm{~min}$
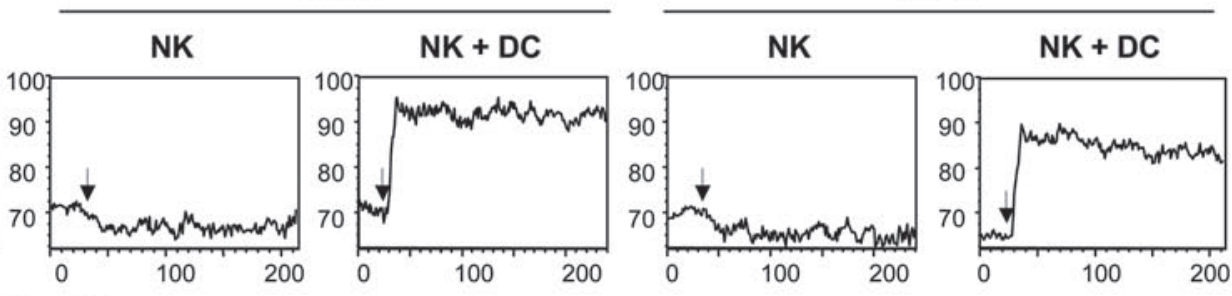

Figure 2

Time (s)

Cell-to-cell contact between NK cells and mDCs was established quickly and resulted in intracellular calcium release. (A and B) NK cells were conjugated by mixing them with $\mathrm{mDCs}$ for $1-5 \mathrm{~min}$ at $37^{\circ} \mathrm{C}$. Cells were stained with anti-LFA-1 and anti-talin mAbs followed by Alexa Fluor 488-conjugated rabbit anti-mouse IgG (green). Shown are LFA-1 and talin in unconjugated resting NK cells (A) and in DC/NK cell conjugates (B) after $1 \mathrm{~min}$ of interaction. White arrows denote DC/NK cell interface. Representative images from 3 experiments are shown. Scale bars: 5 $\mu \mathrm{m}$. (C) Mobilization of intracellular calcium was monitored by flow cytometry. Basal [Ca $\left.{ }^{2+}\right]$ was recorded during $30 \mathrm{~s}$, after which indo-1-loaded $\mathrm{NK}$ cells and $\mathrm{mDCs}$ were mixed with each other for $1 \mathrm{~min}$ at $37^{\circ} \mathrm{C}$ and changes in the fluorescence of NK cells were monitored. NK cells were gated on size, $\mathrm{CD}^{-}$, and CD56right. Black arrows indicate when flow acquisition was appended after formation of conjugates and incubation of cells at $37^{\circ} \mathrm{C}$. Data show the mean value of the response and are representative of 3 experiments. 
A
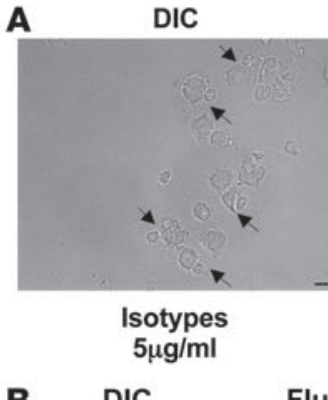

B
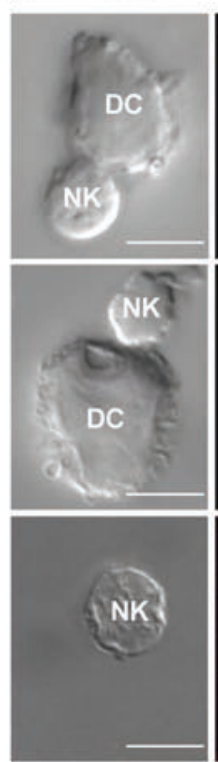

C

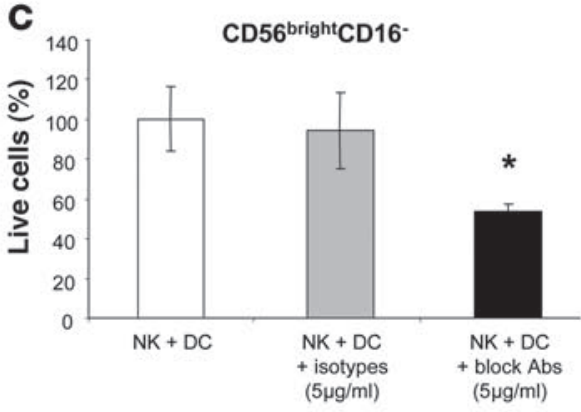

DIC

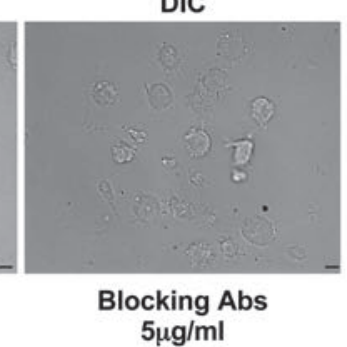

Fluo
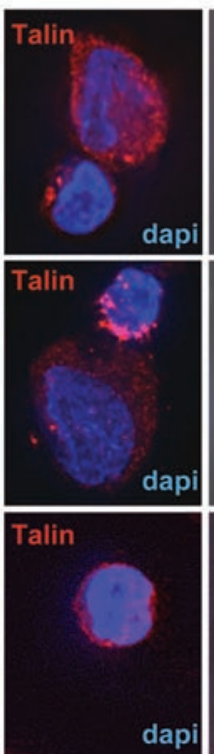

.

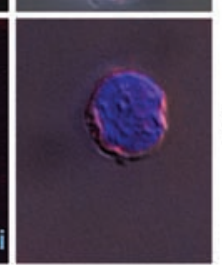

overlay
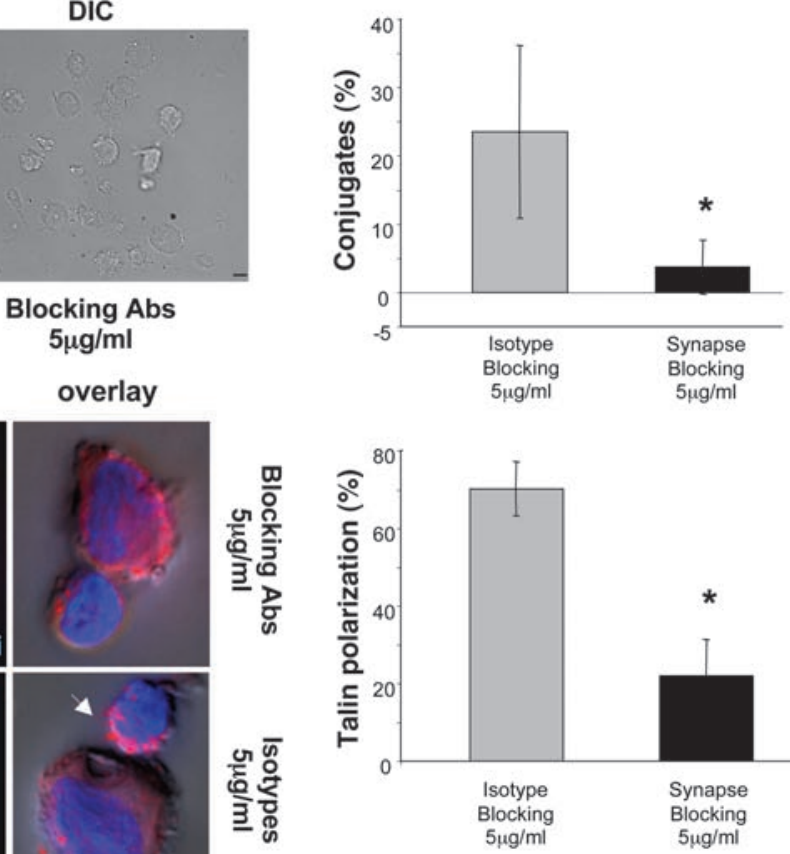
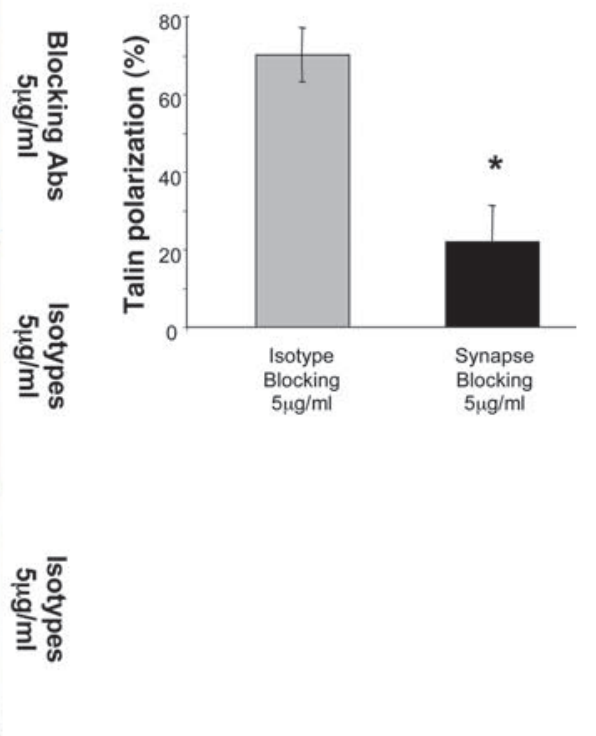

\section{Figure 3}

Synapse formation was necessary for NK cell survival. Poly(l:C)-matured DCs were treated for $2 \mathrm{~h}$ with a mixture of anti-LFA-3, anti-ICAM-1, anti-ICAM-2, and anti-ICAM-3 Abs or control goat IgGs, mouse IgG1, and IgG2b, after which the cells were conjugated or cocultured with purified NK cells. (A and B) DC/NK cell conjugates were formed, fixed, and stained with polyclonal anti-talin followed by Alexa Fluor 555-conjugated donkey anti-rabbit IgG (red). (A) Blocking of adhesion molecules on mDCs in the formation of conjugates (black arrows) and (B) polarization of talin was analyzed. White arrows denote DC/NK cell interface. Representative data from at least 3 different experiments are shown. $n=299$ conjugates (isotype controls); 261 conjugates (synapse blocking). Results are mean \pm SD. ${ }^{*} P<0.01$ versus isotype control. Scale bars: $5 \mu \mathrm{m}$. (C) After 9 days in coculture with DCs in the presence or absence of the indicated Abs, the total cell number of NK cells was analyzed. Results (mean $\pm S D$ ) show the percentage of live cells of at least 3 experiments ran in triplicate. ${ }^{\star} P<0.001$ versus isotype control. NK cells were gated on size, $7 A^{-} D^{-}, C D^{-}$, CD16 ${ }^{+/}$, and CD56 dim/bright.

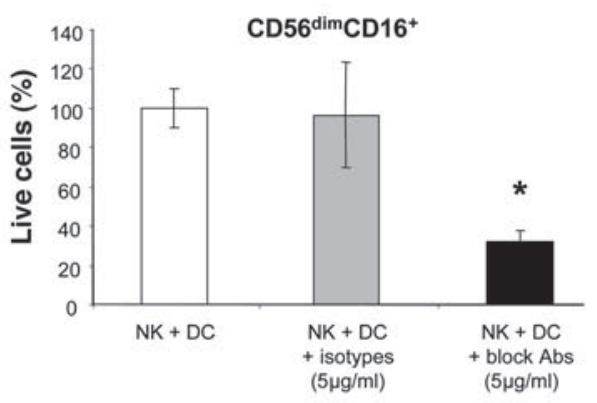

resting NK cells in short-term coculture and conjugation assays. Purified $\mathrm{NK}$ cells, interacting with $\mathrm{mDCs}$, upregulated the early activation marker CD69 after only 20-24 h in coculture, whereas the majority of purified NK cells cultured with DCconditioned medium did not become $\mathrm{CD} 9^{+}$(Figure 1). The percentage of $\mathrm{CD}^{\circ} 9^{+}$activated $\mathrm{NK}$ cells responding to $\mathrm{mDCs}$ was decreased when the cells were separated by a semipermeable membrane in transwell assays. CD69

data suggest that $\mathrm{mDC}$ form a regulatory synapse with resting NK cells, which cannot be readily classified as SMACs or SMICs, and forms the basis of optimal NK cell activation by DCs during innate immune responses.

\section{Results}

Activation of NK cells by DCs required fast-establishing interactions. For the analysis of the immunological synapse formation between human NK cells and DCs, we used monocyte-derived DCs that had been matured in the most efficient way for NK cell activation. We had previously determined that polyinosine-polycytidylic acid-matured [poly(I:C)-matured] DCs stimulated more IFN- $\gamma$ secretion, proliferation, and gain in cytotoxicity of NK cells than LPS or cytokinematured DCs (T. Strowig, unpublished observations). Therefore, poly(I:C)-matured DCs were tested for their capacity to activate was rapidly upregulated and was detected as early as $3 \mathrm{~h}$ after the start of coculture (data not shown). These results suggested that rapid CD69 upregulation on NK cells depends on cellular contact with poly(I:C)-matured DCs (Figure 1).

NK cell activation by mDCs is thought to be dependant, at least in part, on cell-to-cell contact, but the orchestration of rearrangements of involved molecules and kinetics of these interactions have not been characterized to date. Talin and LFA-1 polarization at the interface of cytolytic and noncytolytic conjugates between NK cells and their target cells occurs within $1 \mathrm{~min}$ and is considered to be a very early event in conjugate formation. To further investigate the kinetics of DC/NK cell interactions, we analyzed the segregation of talin and LFA-1 in DC/NK cell conjugates by 2-dimensional immunofluorescence microscopy after 1, 5, and 10 min. In resting NK cells unconjugated with $\mathrm{mDCs}$, both talin 


\section{Table 1}

Polarization of molecules after DC/NK cell and target cell/NK cell interactions

$\begin{array}{lc}\text { Antigen } & \begin{array}{c}\text { Polarized antigen in } \\ \text { DC/NK cell conjugates }(\boldsymbol{n})\end{array} \\ & 72.4 \% \pm 1.3 \%(108) \\ \text { LFA-1A }^{\mathrm{A}} & 73 \% \pm 0.75 \%(141) \\ \text { Talin }^{\mathrm{A}} & 3.3 \% \pm 0.12 \%(110) \\ \text { F-actin }^{\mathrm{B}} & 15.8 \% \pm 5.76 \%(289) \\ \text { Perforin }^{\mathrm{B}} & 77.9 \% \pm 3.22 \%(274) \\ \text { MHC class IC } & 73.8 \% \pm 2.63 \%(192) \\ \text { CD94 } & 65.9 \% \pm 8.93 \%(53) \\ \text { KIRs } & 62.7 \% \pm 1.3 \%(141) \\ \text { IL-15R } \alpha^{\mathrm{D}} & 76.8 \% \pm 2.20 \%(202) \\ \text { IL-15R } \beta & \end{array}$

Polarized antigen in
single NK cells or DCs $(\boldsymbol{n})$
$7.2 \% \pm 3.14 \%(337)$
$7.5 \% \pm 2.26 \%(319)$
NA
NA
$15.4 \% \pm 4.65 \%(273)$
$11.7 \% \pm 2.50 \%(317)$
$15.6 \% \pm 8.63 \%(69)$
$5.7 \%+1.13 \%(319)$
$15.4 \% \pm 4.65 \%(273)$

Polarized antigen in
LCL721.221/NK cell conjugates $(\boldsymbol{n})$
NA
NA
$65.3 \% \pm 4.76 \%(86)$
$65.7 \% \pm 7.04 \%(89)$
NA
$25.9 \% \pm 0.70 \%(166)$
$2.9 \% \pm 2.51 \%(87)$
NA
NA

Polarized antigen in
single activated NK cells $(\boldsymbol{n})$
NA
NA
$4.3 \% \pm 1.86 \%(91)$
$34.2 \% \pm 10.6 \%(93)$
NA
NA
NA
NA
NA

Unconjugated resting or IL-2-activated NK cells, mDCs, LCL721.221 target cells, and DC/NK cell or LCL721.221/NK cell conjugates were formed, fixed, and stained with different Abs. Conjugates or single cells with segregated molecules were counted after 2-dimensional analysis, and results were expressed as percentages. Results from 3 different experiments are shown. AResults are shown for 1 min of interaction between NK cells and mDCs. ${ }^{B}$ Results are shown for $1 \mathrm{~min}$ of interaction between IL-2-activated NK and LCL721.221 cells. ${ }^{C}$ Results concern $\mathrm{mDCs}$ in DC/NK cell conjugates or mDCs as single cells. DPercentages were calculated from double-stained cells with talin at $1 \mathrm{~min}$.

and LFA-1 were evenly distributed at the cell surface after 1 min of incubation (Figure $2 \mathrm{~A}$ ) and remained unpolarized at $5 \mathrm{~min}$ (data not shown). Only approximately $7.5 \%$ of unconjugated NK cells presented a segregation of talin and LFA-1 to one side of the cellular membrane (data not shown). When resting NK cells formed conjugates with $\mathrm{mDCs}, 72.4 \% \pm 1.30 \%$ and $73 \% \pm 0.75 \%$ of conjugates recruited LFA- 1 and talin, respectively, at the contact area within 1 min of coculture (Figure $2 \mathrm{~B}$ ), whereas polarization of both molecules increased to $84.2 \% \pm 3.68 \%$ (LFA- 1 ) and $87.6 \% \pm 1.06 \%$ (talin) after $5 \mathrm{~min}$ of conjugation and stabilized at this level afterward (data not shown).

To determine whether cell-to-cell contacts trigger functional changes in resting NK cells at these early time points (1 and $5 \mathrm{~min}$ ), we loaded NK cells with the ratiometric $\mathrm{Ca}^{2+}$ indicator INDO-1-AM prior to conjugation with mDCs. Basal levels of $\left[\mathrm{Ca}^{2+}\right]$ in resting NK cells were recorded by flow cytometry for $30 \mathrm{~s}, \mathrm{mDCs}$ were added, and conjugates were formed and incubated at $37^{\circ} \mathrm{C}$ for 1,6 , or 8 min. $\mathrm{A} \mathrm{Ca}^{2+}$ rise was observed in resting NK cells within $1 \mathrm{~min}$ after interaction with mDCs (Figure 2C) and was maintained for $4 \mathrm{~min}$, whereas no change in $\left[\mathrm{Ca}^{2+}\right]$ was detected in controls (NK cells alone). Calcium mobilization was also detected after $6 \mathrm{~min}$ (Figure 2C) and 8 min of conjugation (data not shown). These data show that functional DC/NK cell interactions are already established within the first 5 minutes of coculture, and we used this early time window to characterize the composition of the cellular interactions.

The formation of a functional conjugate was necessary for NK cell survival. The importance of adhesion molecules such as LFA-1, and to a lesser extend LFA-3, and their polarization to the cellular interface for synapse formation has been shown previously (35). We decided to prevent the conjugate formation between NK cells and mDCs using blocking Abs against LFA-1 ligands, i.e., ICAM-1, ICAM-2, ICAM-3, and LFA-3. We first checked whether those Abs inhibited the early events detectable after DC/NK cell interactions. When compared with the control conditions, pretreatment of mDCs with adhesion molecule-blocking Abs significantly decreased the formation of conjugates within the first $5 \mathrm{~min}$ of encounter (Figure 3A). We also observed significantly lower talin polarization at the cellular junction between NK cells and mDCs after adhesion molecule blockade, whereas isotype-treated conjugates had talin recruitment comparable to that of untreated conjugates
(Figure 3B). Talin was not polarized or segregated in resting NK cells unconjugated with mDCs (Figure $2 \mathrm{~A}$ and Figure $3 \mathrm{~B}$ ). Next, we performed DC/NK cell cocultures in the presence of blocking Abs and evaluated NK cell survival after 9 days. The survival of both subsets of NK cells was significantly reduced, by $40 \%$ for the CD56 $6^{\text {bright }}$ subset $(P<0.001)$ and by $60 \%$ for the CD56 $6^{\text {dim }}$ subpopulation $(P<0.001)$, when adhesion-blocking Abs were added (Figure $3 C)$. Thus, the synapse establishment via LFA-1- and LFA-3-mediated conjugate formation is essential in NK cell survival during interactions with $\mathrm{mDCs}$.

Early interactions between resting $N K$ cells and $m D C$ displayed hallmarks of a supramolecular inhibitory complex. Interactions between NK cells and autologous target cells of hematopoietic origin are usually dominated by the inhibitory signals preventing cytotoxicity (3). However, resting NK cells are activated by interactions with autologous $\mathrm{mDCs}$, yet lysis of $\mathrm{mDCs}$ does not occur after interaction. In order to discriminate between inhibitory and activating NK cell interactions, we used markers differentially recruited at the contact area in cytolytic and noncytolytic interactions (Table 1). As shown in Figure 4, although a minority of $15.9 \% \pm 5.76 \%$ of conjugates showed clustered perforin at the DC/NK cell interface, perforin was not recruited to the contact area in the majority of DC/NK cell conjugates from $1 \mathrm{~min}$ to $5 \mathrm{~min}$ after conjugation in contrast to cytolytic interactions. We also did not find any sign of polarization of F-actin in DC/NK cell conjugates within 1-5 min of conjugations, with only $3.3 \% \pm 0.12 \%$ of conjugates positive for a segregated F-actin at the interface. These findings indicate that hallmarks of cytolytic interactions, which are found at the interface between NK cells with susceptible target cells, were absent in $\mathrm{DC} / \mathrm{NK}$ cell interactions.

KIRs and the CD94/NKG2A receptor bind to MHC class I molecules on the surface of target cells to trigger inhibitory signaling within NK cells. Interestingly, the C-type lectin CD94 and the Igtype receptors KIR2DL1/DL2 were enriched at the DC/NK cell interface very quickly after conjugation. After $1 \mathrm{~min}, 73.8 \% \pm 2.63 \%$ and $65.9 \% \pm 8.93 \%$ of conjugates had clustered CD94 and KIRs, respectively, whereas $11.7 \% \pm 2.50 \%$ (CD94) and $15.6 \% \pm 8.63 \%$ (KIRs) of unconjugated NK cells presented such clustering. This segregation was sustained for at least $10 \mathrm{~min}$. Since CD94 can also form activating heterodimers with NKG2C and NKG2E/H (36, 


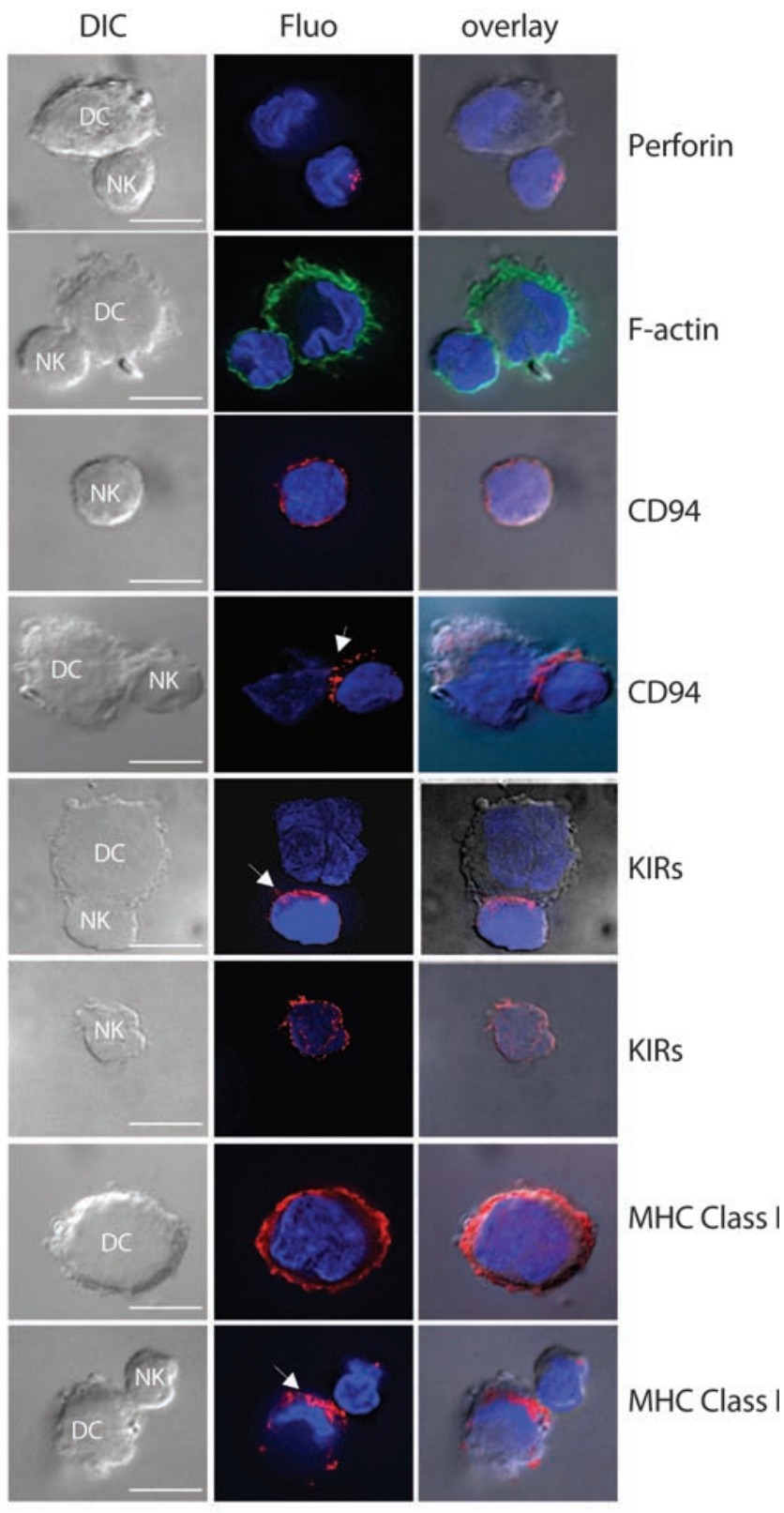

37), we were interested in determining the percentage of NK cells positive for the inhibitory CD94/NKG2A heterodimer. Between 97\% and $99 \%$ of freshly isolated NK cells were positive for both CD94 and NKG2A (data not shown). Because all CD94 molecules polarized to the DC/NK cell interface, we suggest therefore that the inhibitory CD94/NKG2A receptor was also localized to the DC contact site on NK cells. Notably, not all DC/NK cell conjugates displayed KIR polarization, because only a subset of NK cells is positive for the expression of KIRs (38), and only conjugates with KIRpositive NK cells could be evaluated for polarization. In addition, within the same time frame, MHC class I molecules also segregated very rapidly toward the cell-to-cell contact: $77.9 \% \pm 3.22 \%$ of $\mathrm{mDCs}$ conjugated with NK cells polarized MHC class I molecules at the interface, whereas the same clustering on one side of unconjugated mDCs was only observed in $15.4 \% \pm 4.65 \%$ of cells. In contrast to these findings with DC/NK cell synapses, when IL-2-activated NK

\section{Figure 4}

CD94, KIRs, and MHC class I polarized to the cell-to-cell contact area, but perforin and $\mathrm{F}$-actin did not segregate during $\mathrm{DC} / \mathrm{NK}$ cell interactions. Unconjugated mDCs, NK cells, and DC/NK cell conjugates were formed, fixed, and stained with anti-perforin, anti-CD94, anti-KIRs, and anti-HLA-A, -B, and -C mAbs, followed by Alexa Fluor 555-conjugated rabbit anti-mouse IgG (red). F-actin was stained with bodipy-conjugated phallocidin (green). White arrows denote DC/NK cell interface. Images are representative of evaluation of 100-200 conjugates from 3 experiments. Scale bars: $5 \mu \mathrm{m}$.

cells were conjugated with LCL721.221 target cells, which are susceptible to NK cell lysis, the 2-dimensional analysis of the interphase revealed a typical activating and cytotoxic synapse, with polarization of perforin and F-actin, and absence of segregation of CD94 and KIRs (Table 1 and Supplemental Figure 1; supplemental material available online with this article; doi:10.1172/JCI31751DS1). In DC/NK cell conjugates as well as LCL721.221/NK cell conjugates, amounts of molecules that redistributed to the DC/NK cell contact site were quantified. The fluorescence of all molecules segregated at the interface in conjugates was significantly increased by at least 2 -fold compared with the fluorescence in unconjugated cells, suggesting a real translocation of molecules during conjugation with mDCs or target cells (Table 2 and Supplemental Table 1). Thus, data obtained after DC/NK cell interactions were consistent with the establishment of noncytolytic interaction.

IL-15R $\alpha$ was polarized at the interface of DC/NK cell conjugates. Despite the inhibitory buildup of the DC/NK cell synapse, mDCs activate resting NK cells to secrete cytokines, to enhance cytotoxicity, to survive, and to proliferate. IL-15 has been shown to be involved in the activation of NK cells $(39,40)$, particularly in promoting proliferation, as well as in NK cell survival (24). Therefore, we analyzed IL-15R distribution at the DC/NK cell interface. The highly specific receptor subunit $\alpha$ has a wide cellular distribution (41). Thus, we first characterized expression of IL-15R $\alpha$ in our system. Poly(I:C)matured DCs expressed IL-15R $\alpha$ (Figure 5A) at higher levels than did immature DCs (imDCs). Blood NK cells also expressed intermediate levels of IL-15R $\alpha$ (Figure 5, B and C). Notably, analyses of mean fluorescence intensities after flow cytometric IL-15R $\alpha$ staining with and without permeabilization showed that intracellular IL-15R $\alpha$ was 18 -fold more abundant than extracellular IL-15R $\alpha$ in resting unconjugated NK cells (Supplemental Figure 2). We also verified IL-15R $\alpha$ expression by Western blot analysis in these 3 different cell types, and our analysis revealed similar levels of total IL-15R $\alpha$ in NK cells, mDCs, and imDCs (Figure 5C). Next, we investigated the localization of IL-15R $\alpha$ during DC/NK cell interactions. IL-15R $\alpha$ was polarized to the cell-to-cell contact area in 2-dimensional analysis of conjugates at $1 \mathrm{~min}$ and remained polarized in the conjugates when analyzed over the next $10 \mathrm{~min}$ (Figure 5D and data not shown). As shown in Figure 5E, the clusters of IL-15R $\alpha$ polarized at the interface were localized within Cell Trace-labeled NK cells, whereas no selective segregation of the IL-15R $\alpha$ chain toward the DC/NK cell interface was observed at these time points in mDCs. Similar polarization of IL-12, IL-15, or DC-SIGN was not observed within the first $10 \mathrm{~min}$ of DC/NK cell conjugate formation (data not shown). The basal level of IL-15R $\alpha$ polarization in resting NK cells was $5.7 \% \pm 1.13 \%$ (Table 1 ). Approximately $60 \%$ of conjugates presented IL-15R $\alpha$ recruitment to the contact area at 1 min after conjugation, suggesting a rapid formation of the complex. Because the IL-15R $\alpha$ and $\beta$ chains complex to form the high- 


\section{Table 2}

Quantification of molecule polarization after DC/NK cell and target cell/NK cell interactions

\begin{tabular}{lcc}
\hline & \multicolumn{2}{c}{ RE synapse/RE opposite membrane ratio } \\
Antigen & Conjugate & Single cells \\
LFA-1 $^{\mathrm{B}}$ & $231.9 \% \pm 56.20 \%$ & $100 \% \pm 18.47 \%$ \\
Talin $^{B}$ & $273.6 \% \pm 56.20 \%$ & $100 \% \pm 14.23 \%$ \\
MHC class IC & $261.3 \% \pm 31.99 \%$ & $100 \% \pm 25.01 \%$ \\
KIRs & $300.2 \% \pm 58.78 \%$ & $100 \% \pm 13.22 \%$ \\
CD94 & $190.1 \% \pm 29.28 \%$ & $100 \% \pm 11.46 \%$ \\
IL-15R $\alpha$ & $202.6 \% \pm 46.18 \%$ & $100 \% \pm 18.42 \%$ \\
IL-15R 3 & $221.4 \% \pm 59.98 \%$ & $100 \% \pm 17.31 \%$
\end{tabular}

Unconjugated NK cells, mDCs, and DC/NK cell conjugates were formed, fixed, and stained with different Abs. Values for conjugates were normalized and transformed into percentages relative to values from single cells, assigned as $100 \%$. Results from 3 different experiments are shown $(n=10-15) . P<0.001$ versus unconjugated controls

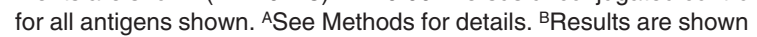
for $1 \mathrm{~min}$ of interaction between NK cells and mDCs. ${ }^{C}$ Results concern $\mathrm{mDC}$ in $\mathrm{DC} / \mathrm{NK}$ cell conjugates or $\mathrm{mDCs}$ as single cells.

affinity cytokine receptor (32), we also determined the localization of IL-15R $\beta$. In conjugates analyzed at $1 \mathrm{~min}$ of interaction, IL-15R $\beta$ was enriched at the DC/NK cell interface at levels comparable to those of IL-15R $\alpha$ (Figure 5F and Table 1). These data show that IL-15R polarizes within NK cells to the cell-to-cell contact area.

Activating and inhibitory interactions were enriched in the central SMIC of the DC/NK cell synapse. Because our 2-dimensional analysis of DC/NK cell conjugates demonstrated that IL-15R, CD94, KIRs, talin, and LFA-1 polarized with similar kinetics, we determined where these molecules were localized in the 3-dimensional NKIS. In addition, we determined whether receptors for activating signals (such as IL-15R $\alpha$ ) and inhibitory signals (such as CD94) are confined to different areas of the central synapse in order to trigger opposite signals in parallel. We conjugated cells and stained for IL-15R $\alpha$, CD94, KIRs, talin, or LFA-1, and the spatial distribution of the molecules was determined. After $1 \mathrm{~min}$, LFA-1, talin, CD94, KIRs, and IL-15R $\alpha$ showed similar segregation patterns at the contact area between DCs and NK cells in 2-dimensional analysis (Figure 6A). IL-15R $\alpha$ was always polarized together with LFA- 1 and talin at the DC/NK cell interface (Figure 6B). The NKIS at 1 min displayed segregation of LFA-1 and talin at the periphery of the SMAC or SMIC, whereas IL-15R $\alpha$, CD94, and KIRs appeared as a more central cluster surrounded by LFA-1 or talin (Figure 6C). Similar results were observed after $5 \mathrm{~min}$ of conjugation (data not shown). Therefore, IL-15R $\alpha, \mathrm{CD} 94$, and KIRs localized to the center, while talin and LFA-1 were found in the periphery of the DC/NK cell synapse. When we colabeled for IL-15R $\alpha$ and CD94 or IL-15R $\alpha$ and KIRs, 3-dimensional analyses showed distinct areas for IL-15R $\alpha$ and CD94 as well as IL-15R $\alpha$ and KIRs, with little overlap in the synapse center (Figure 6C). As shown in Figure 6D, IL-15R $\alpha$ and CD94 shared only $8.8 \% \pm 7.65 \%$, and IL- $15 \mathrm{R} \alpha$ and KIRs only $8.42 \% \pm 5.3 \%$, of voxels at the reconstructed synapse, whereas the percentage of voxels colocalized for the single IL-15R $\alpha$ and CD94 signals compared with the respective total number of voxels was $24.2 \% \pm 17.81 \%$ and $17.9 \% \pm 13.84 \%$, respectively, and $18.78 \% \pm 11.03 \%$ and $15.54 \% \pm 107 \%$ for IL-15R $\alpha$ and KIRs, respectively. This suggested that activating and inhibitory signals are transmitted from different areas of the central DC/NK cell synapse, allowing initiation of activating and inhibitory interactions in parallel.

$m D C$ preferentially formed conjugates with $C D 56^{\text {bright }} C D 16^{-} \mathrm{NK}$ cells. The minor blood NK subset of CD56 $6^{\text {bright }} \mathrm{CD} 16^{-}$cells has previously been shown to interact preferentially with $\operatorname{mDCs}(17,24)$ (T. Strowig, unpublished observations). We sorted blood NK cells into the 2 main subpopulations (CD56 ${ }^{\mathrm{dim}} \mathrm{CD} 16^{+}$and $\mathrm{CD} 56^{\text {bright }} \mathrm{CD} 16^{-}$) and conjugated $\mathrm{mDCs}$ with both subsets at identical ratios and at normalized ratios representing the percentages of both subpopulations in blood (95\% to 5\%, respectively). At identical ratios, conjugation between $\mathrm{CD} 56^{\text {bright }} \mathrm{CD} 16^{-}$cells and mDCs reached levels of conjugation observed when total blood NK cells interacted with $\mathrm{mDCs}(47.2 \% \pm 15.56 \%$ and $45.0 \% \pm 16.54 \%$, respectively; Figure 7), whereas $\mathrm{CD} 56^{\mathrm{dim}} \mathrm{CD} 16^{+} \mathrm{NK}$ cells formed significantly fewer conjugates with $\mathrm{mDCs}$, i.e., at levels comparable to those of total resting imDC/NK cell conjugates $(21.55 \% \pm 9.10 \%$ and $10.6 \% \pm 11.86 \%$, respectively). Strikingly, percentages of conjugates between $\mathrm{CD} 56^{\mathrm{dim}} \mathrm{CD} 16^{+} \mathrm{NK}$ cells and $\mathrm{mDCs}$ became similar only when the ratio of these NK cells was increased by 20 -fold before interaction $(48.45 \pm 4.71 \%)$ in order to respect the ratio of NK cell subsets in blood (data not shown). In agreement with our previous data, conjugates between both subtypes of NK cells and mDCs polarized IL-15R $\alpha$ at the cell-to-cell contact site within $1 \mathrm{~min}$ after interaction (data not shown). These data suggest that immunoregulatory CD56 $6^{\text {bright }} \mathrm{CD} 16^{-} \mathrm{NK}$ cells form conjugates with $\mathrm{mDCs}$ more readily than with cytotoxic $\mathrm{CD} 56^{\mathrm{dim}} \mathrm{CD} 16^{+} \mathrm{NK}$ cells.

Polarized IL-15R $\alpha$-mediated NK cell survival in DC/NK cell conjugates. The polarization of IL-15R $\alpha$ at the NKIS raised the question of whether the enrichment has a functional relevance for NK cells. Therefore, we blocked IL-15R $\alpha$ and analyzed the survival of NK cells in cocultures. Pretreatment of NK cells with the blocking anti-IL-15R $\alpha \mathrm{Ab}$ decreased the percentage of live NK cells after 9 days in cocultures with mDCs $(P<0.01$; Figure $8 \mathrm{~A})$. Live cell numbers were significantly decreased in both main blood NK cell subpopulations $(P<0.01$; Figure $8 \mathrm{~B})$. To determine the percentages of apoptotic cells in blocking IL-15R $\alpha \mathrm{Ab}$ - and isotype-treated NK cell cocultures with DCs, we performed 6-color flow cytometric analysis with annexin V. Compared with control cultures, antiIL-15R $\alpha$-treated live CD56 $6^{\text {bright }} \mathrm{CD} 16^{-}$and CD56 ${ }^{\text {dim }} \mathrm{CD} 16^{+} \mathrm{NK}$ cells $\left(7 \mathrm{AAD}^{-}\right)$showed upregulation of annexin $\mathrm{V}$ binding at the cell surface $\left(60 \%\right.$ and $84 \%$ of annexin $\mathrm{V}^{+}$cells, respectively; $P<0.02$; Figure $8, \mathrm{C}$ and $\mathrm{D})$, suggesting that IL-15R $\alpha$ plays a role in protection of NK cells from apoptosis.

Recently, it has been shown that NK and T cells from patients with infectious mononucleosis (IM) lack expression of IL-15R $\alpha$ on their surface and maintain this loss for up to 14 years after IM, while normal levels of IL-15R $\alpha$ can be detected on their B cells and monocytes (42). Therefore, we used PBMCs from healthy individuals with a history of IM to address the influence of IL-15R $\alpha$ on NK cells for their survival upon coculture with DCs. Consistent with previous observations, we found diminished IL-15R $\alpha$ expression on NK cells from former IM patients compared with healthy individuals with no IM history (Figure 9A). Normal levels of expression were observed on mDCs derived from monocytes of individuals that had experienced IM (Figure 9B). Because of the lack of IL-15R $\alpha$ on NK cells from donors with $\mathrm{IM}$, no polarization of the receptor in NK cells during the first $2 \mathrm{~min}$ of conjugate formation between NK cells and mDCs was observed (Figure 9B). Next, we performed DC/NK cell cocultures. As shown in Figure 9C, ratios between output and input of total NK cells numbers differed significantly between individuals with and without prior IM 

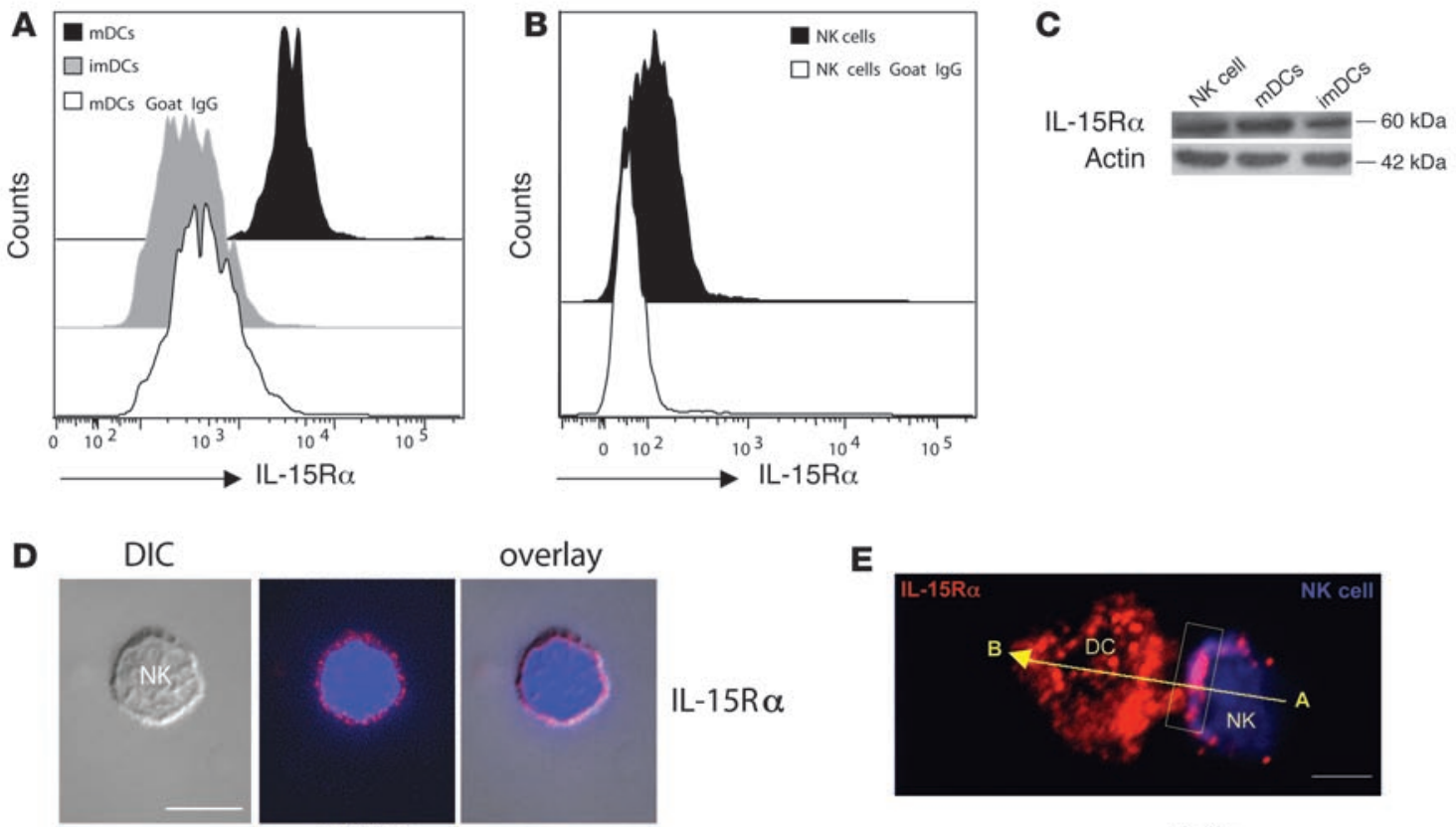

IL-15R $\alpha$

E
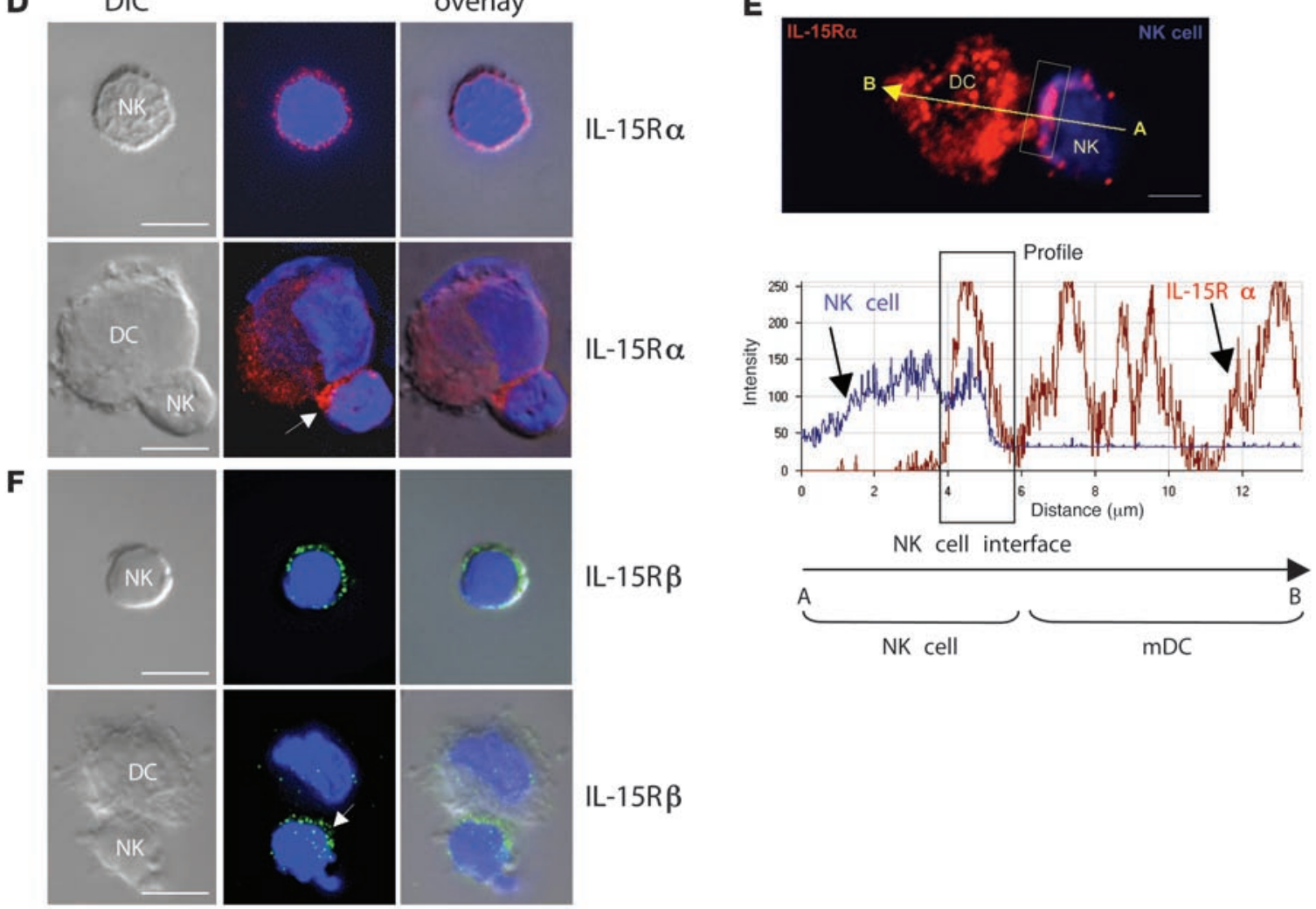

\section{Figure 5}

IL-15R $\alpha$ was expressed by mDCs and NK cells, and IL-15R $\alpha$ and IL-15R $\beta$ accumulated, at the interface of NK cells with mDCs. (A and B) imDCs and mDCs (A) and purified blood NK cells (B) were washed, stained with anti-IL-15R $\alpha$ polyclonal Ab followed by Alexa Fluor 488- or Alexa Fluor 647-conjugated donkey anti-goat IgG, and analyzed by flow cytometry. DCs were gated on size, CD11 $\mathrm{c}^{+}$, and CD83 (CD83+ for mDCs, $\mathrm{CD}^{-} 3^{-}$for imDCs); NK cells were gated on size, CD3-, and CD56 ${ }^{+}$. Representative data from 3 experiments are shown. (C) Cells were also lysed in buffer containing $1 \%$ NP-40, and proteins were separated by SDS-PAGE. IL-15R $\alpha$ was then visualized by Western blotting with anti-IL-15R $\alpha$ $\mathrm{mAb}$ followed by a goat anti-mouse-HRP secondary antiserum. Data are representative of 3 separate experiments. Immunofluorescence was performed with polyclonal anti-IL-15R $\alpha$ followed by Rhodamine Red-X-conjugated donkey anti-goat IgG (red) or with IL-15R $\beta$ mAb followed by Alexa Fluor 488-conjugated rabbit anti-mouse IgG (green). (D) IL-15R $\alpha$ localization in unconjugated resting NK cells and DC/NK cell conjugates after 1 min of interactions. (E) Fluorescence intensities of IL-15R $\alpha$ (red) and Cell Trace Far Red DDAO-SE-labeled NK cells (pseudocolored in blue) were plotted (bottom) along the trajectory illustrated above. The area of the synapse on the NK cell side is delimited by boxes. (F) Images of IL-15R $\beta$ localization in unconjugated resting NK cells and DC/NK cell conjugates after 1 min of DC/NK cell coculture. White arrows denote DC/NK cell interface. Results are representative of 100-200 conjugates in at least 3 separate experiments. Scale bars: $5 \mu \mathrm{m}$.

history. DCs were less efficient to rescue survival of NK cells from IM donors, suggesting that the presence of IL-15R $\alpha$ plays a role on NK cell survival after interactions with mDCs. Additionally, compared with NK cells of donors without prior IM history, blocking of IL-15R $\alpha$ in cultures from former IM patients did not significantly reduce NK cell survival, while IL-15R $\alpha^{+} \mathrm{NK}$ cell survival was significant decreased by this treatment (Figure 9D). These data indicate that IL-15R $\alpha$ on NK cells contributes to NK cell survival after activation by $\mathrm{mDCs}$. 
A DIC
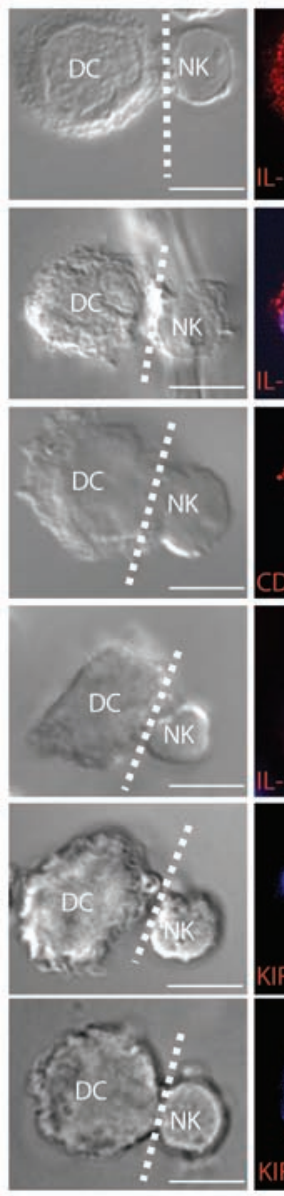

Fluo
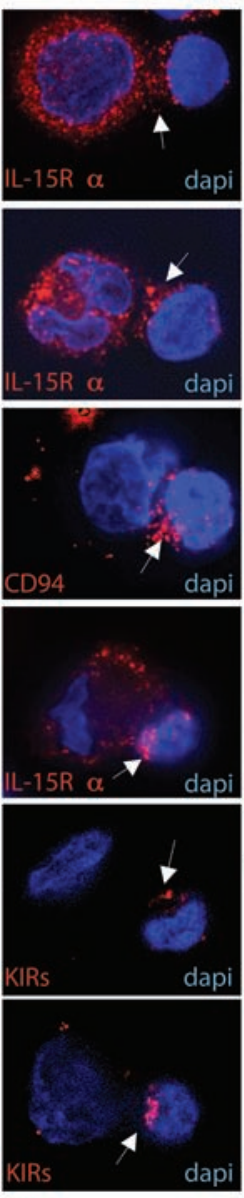

C Z-plane

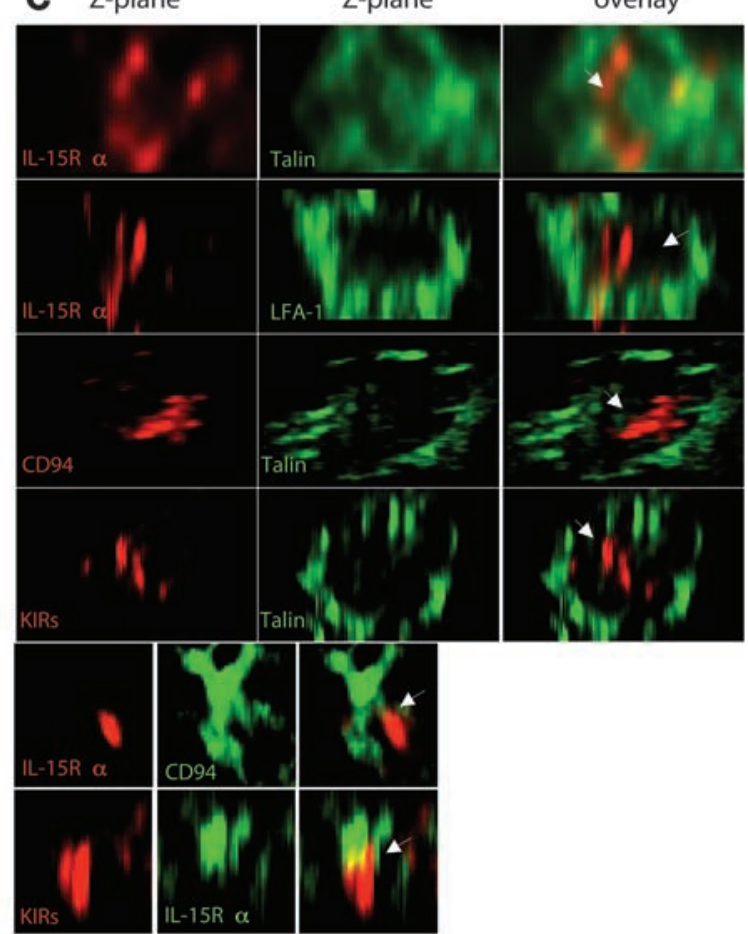

Fluo
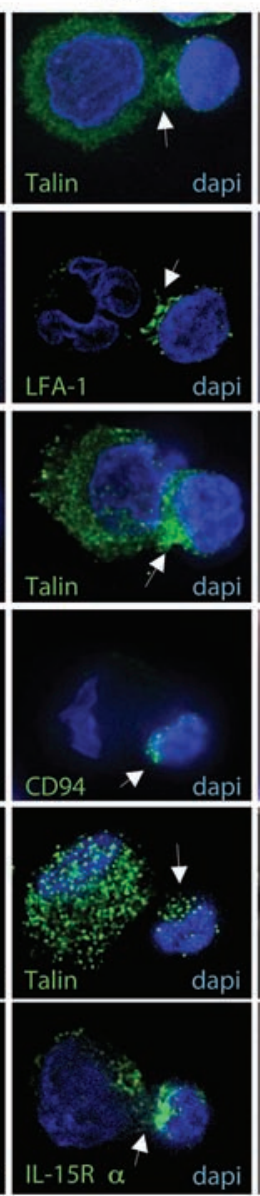

dapi
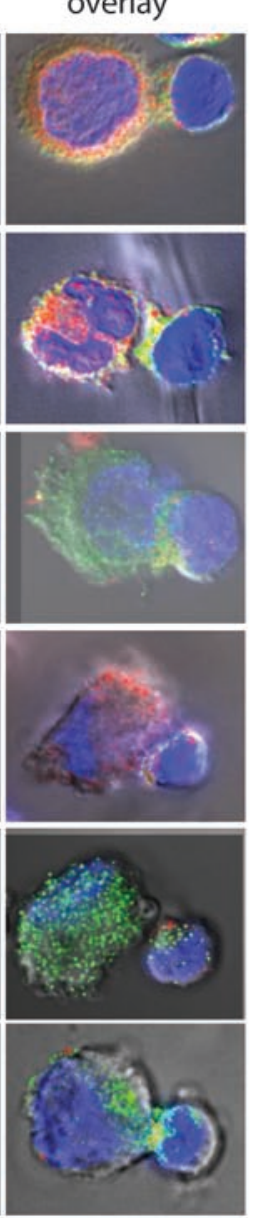

D
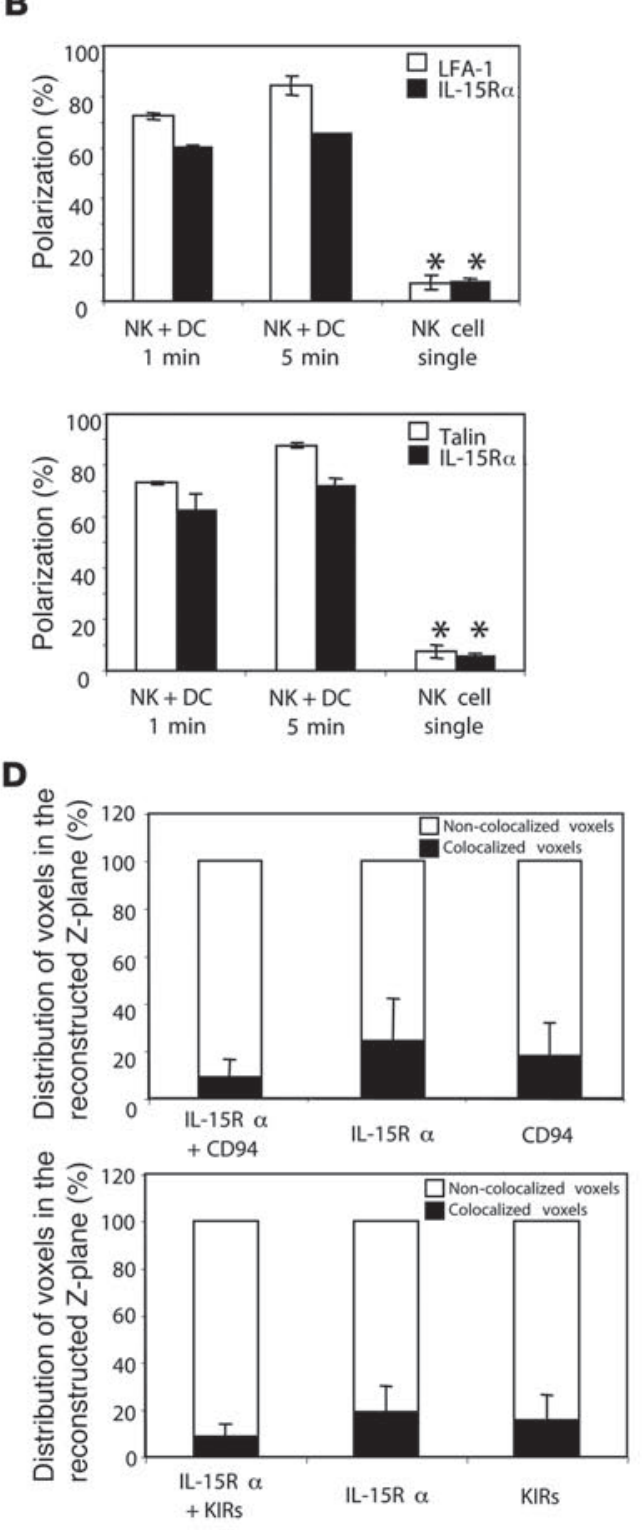

\section{Figure 6}

Survival and inhibition signals were enriched at the immune synapse during NK cell and $\mathrm{mDC}$ interactions. (A) Conjugates were formed, fixed, and colabeled with anti-LFA-1, anti-talin, anti-CD94, anti-KIRs, and antiIL-15R $\alpha$ followed by appropriate secondary Abs. White arrows denote DC/NK cell interface. (B) Quantification of the polarization of IL-15R $\alpha$ compared to polarization of talin and LFA-1 at 1 and 5 min of interactions and in unconjugated resting NK cells (1 min). Means \pm SD are shown, where at least 100 conjugates for each labeling were analyzed in 3 separate experiments. ${ }^{*} P<0.01$ versus $\mathrm{NK}+\mathrm{DC}$ at $1 \mathrm{~min}$. (C) Reconstructed Z-stack contact view of the areas denoted by dotted lines in the DIC images in A. Alexa Fluor 488-labeled LFA-1, talin, and CD94 or rhodamine Red-X-labeled IL-15R $\alpha$ or KIRs are shown. White arrows denote synapse center. (D) Distribution of voxels within the reconstructed Z-plane. Values are expressed as percentage of the total number of voxels from each single channel (CD94/IL-15R $\alpha$ or KIRs/IL-15R $\alpha$ ) or total number of voxels from the overlay image (IL-15R $\alpha+$ CD94 or IL-15R $\alpha+$ KIRs). Shown are 6 reconstructed Z-planes from 3 different experiments. 


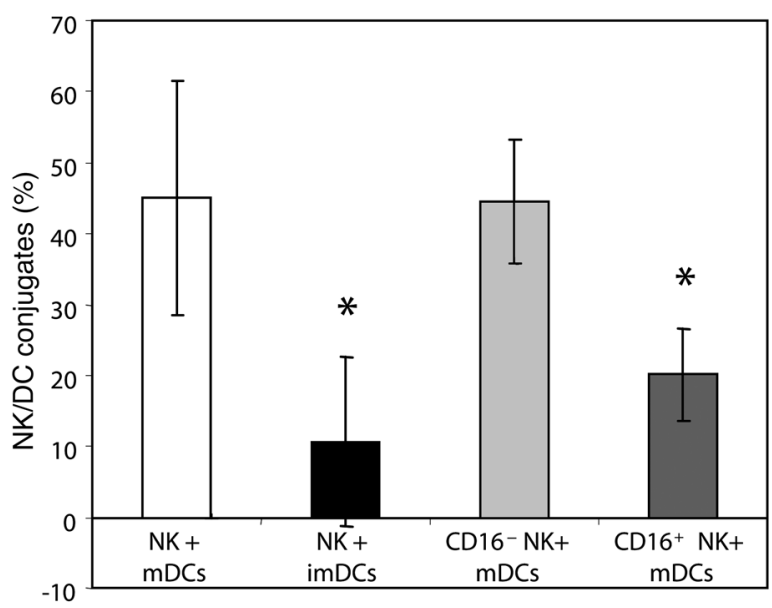

Figure 7

CD56 ${ }^{\text {bright }}$ CD16- NK cells formed more conjugates with mDCs. Percentage of conjugates between CD56 ${ }^{\text {dim }} C D 16^{+}$or CD56 bright $C D 16^{-}$NK cells and mDCs or imDCs. Data are mean \pm SD of 200 NK cells from 5 separate experiments. ${ }^{*} P<0.01$ versus respective control.

\section{Discussion}

NK cells and DCs are central players in the innate immune response, especially during early phases of viral infections (43). In this study, we showed that cell-to-cell contacts with the establishment of an immunological synapse were essential for complete activation of NK cells by mature DCs. Furthermore, the data presented herein suggest that models of synapse formation between target cells and NK cells, discriminating between activating and inhibitory synapses, are not applicable to DC/NK cell interactions. Rather, we propose that DC/NK cell interactions lead to a regulatory synapse formation containing features of inhibitory interactions (i.e., polarized CD94, KIRs, unpolarized perforin and F-actin, and absence of killing), but also displaying classic hallmarks of activation (i.e., intracellular calcium mobilization, sustained physical interactions, and activation of NK cells to upregulate CD69 and to survive via IL-15/IL-15R $\alpha$ interaction). Our data suggest that the regulatory synapse between NK cells and DCs limits optimal NK cell activation to areas of close DC/NK cell encounter like the parafollicular T cell zone of secondary lymphoid organs and sites of inflammation.

We used monocyte-derived DCs as a model of myeloid human DCs in our study. This type of DC has been primarily used in DCbased immunotherapies of tumor patients, and our results elucidate how these DC vaccines can engage NK cells in addition to $\mathrm{T}$ cell stimulation after in vivo infection (44). Monocyte-derived DCs are comparable to splenic and blood myeloid DCs, as well as in vitro-differentiated interstitial-dermal DCs, with respect to NK cell activation $(24,45,46)$. Furthermore, monocyte differentiation into DCs at infection sites might contribute important DC populations for the initiation of immune responses $(44,47)$. This NK cell activation by myeloid DCs depends on both soluble factors and cell-to-cell contacts $(24,48)$ (T. Strowig, unpublished observations), but a role for DC/NK cell synapse formation had not been established in this interaction.

So far, studies on DC/NK cell conjugate formations have mainly relied on 2-dimensional analysis at late time points after conjugation, such as $25 \mathrm{~min}$ or even $3 \mathrm{~h}(33,34)$. These analyses, although interesting, did not take into consideration the fast kinetics and spatiotemporal composition of immunological synapses. Thus, characterization of DC/NK cell synapses at early time points and involving 3-dimensional reconstruction, as performed in the present study, are important, because essential differences between noncytolytic and cytolytic NKIS have been established with similar methods. Indeed, as far as inhibitory synapses are concerned, interactions between MHC class $I^{\text {high }}$ targets and NK cells lead to polarization of talin and LFA-1 at the synapse within 1 min of interaction, but no further downstream signaling is activated and these interactions result in unstable, short-lived, noncytolytic conjugates (9). In noncytolytic conjugates, talin recruitment is transient, because rapid dissociation of talin clusters is observed within $5 \mathrm{~min}$ of inhibitory interactions (5). On the other hand, activating synapses between MHC class I $^{\text {low }}$ cells and NK cells result in sustained recruitment of talin and LFA-1 to the peripheral SMAC, polarization of perforin followed by the killing of target cells, and polymerization of cytoskeletal F-actin at the synapse $(5,12,49)$. In contrast to both of these previously described synapse compositions, interactions between NK cells and MHC class I $^{\text {high }} \mathrm{mDC}$ formed fast and lasted at least $10 \mathrm{~min}$. We observed calcium flux from 1 to $8 \mathrm{~min}$ after DC/NK cell coculture; the majority of conjugates presented segregated LFA- 1 and talin at peripheral SMACs from 1 to $10 \mathrm{~min}$; F-actin and perforin were not polarized; and long-lasting conjugates were observed by videomicroscopy for the duration of 10 min (data not shown). While we did not observe F-actin accumulation at the DC/NK cell synapse at early time points, polarization of F-actin has been seen after 25 min of conjugation between mDCs and NK cells, suggesting that the cytoskeleton remodeling might occur at later time points (33). Lasting interactions have been observed in conjugates between peripheral NK cell lines and NK cell-susceptible target cells, but in those conjugates LFA-1 was localized in central patches of intercellular adhesion (8), instead of being segregated to the periphery of the supramolecular cluster like in our experiments. In addition, we found with CD94 and KIR polarization on NK cells and MHC class I polarization on DCs at the conjugate interfaces, characteristics of inhibitory interactions at the center of the synapse between these 2 cell types. Specifically, these molecules have been shown to mediate protection of mature DCs from NK cell lysis. Namely, mature DCs are protected from NK cell lysis by their increased expression of MHC class I (50), and the CD94/NKG2A receptor primarily mediates this inhibition of DC lysis by NK cells (51). These data suggest that although DC/NK cell synapses display hallmarks of inhibitory NK cell synapses, they are long lasting like activating NK cell synapses.

Interactions between NK cells and mDCs cause rapid accumulation of IL-15R $\alpha$ at the central synapse in NK cells within 1-5 min after DC/NK cell conjugate formation. Because of the limitations of fluorescence imaging, the polarized IL-15R $\alpha$ could be present at the cell surface or might reflect recently engaged receptors that have been internalized or receptors in secretory vesicles that are on their way to the surface. Indeed, recycling from the membrane has been previously observed for IL-15R $\alpha$ (31). However, the significant amount of IL-15R $\alpha$ that was found on the surface of unconjugated NK cells (Supplemental Figure 2) suggests that IL-15R $\alpha$ is also present on the surface of the DC/NK cell interface. We did not observe any accumulation of IL-12 or IL-18 in the cell contact area at these early time points. However, these cytokines were previously found to be polarized in $\mathrm{mDCs}$ at $25 \mathrm{~min}$ (33) and $3 \mathrm{~h}$ (34). Thus, we propose that polarization of IL-15R $\alpha$ in NK cells precedes cytokine polarization in DCs and constitutes an early event 
A
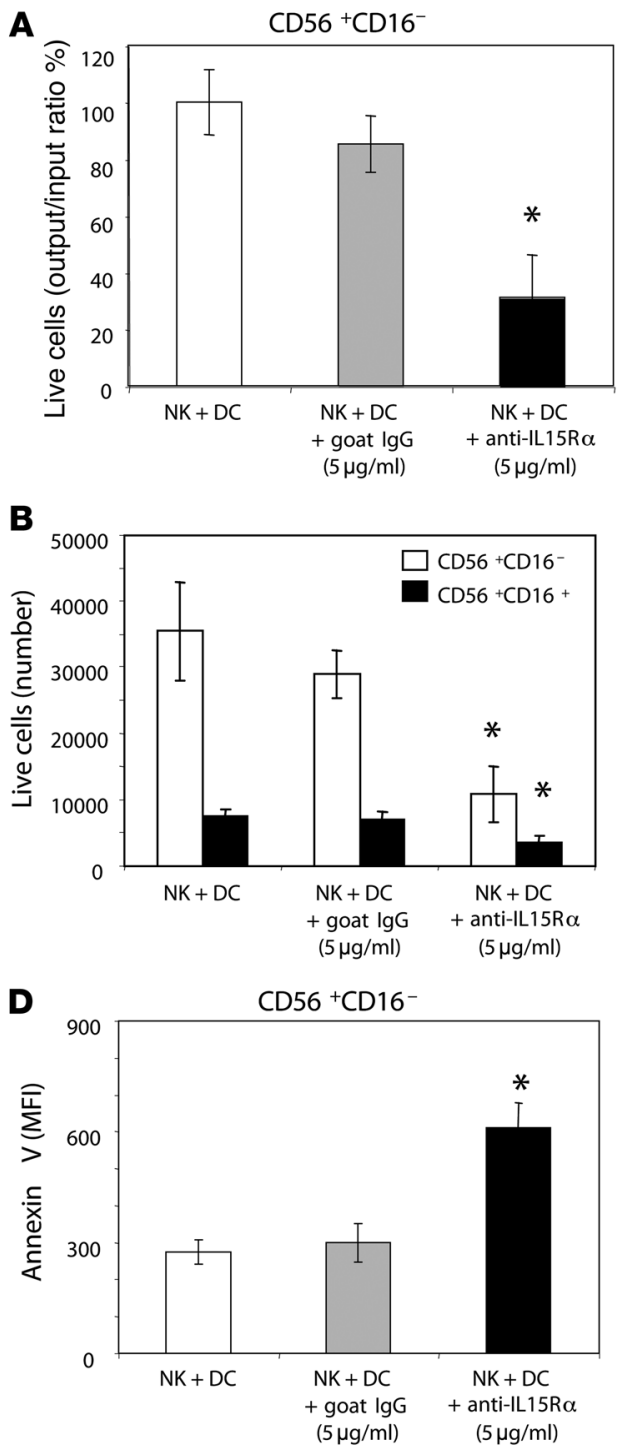
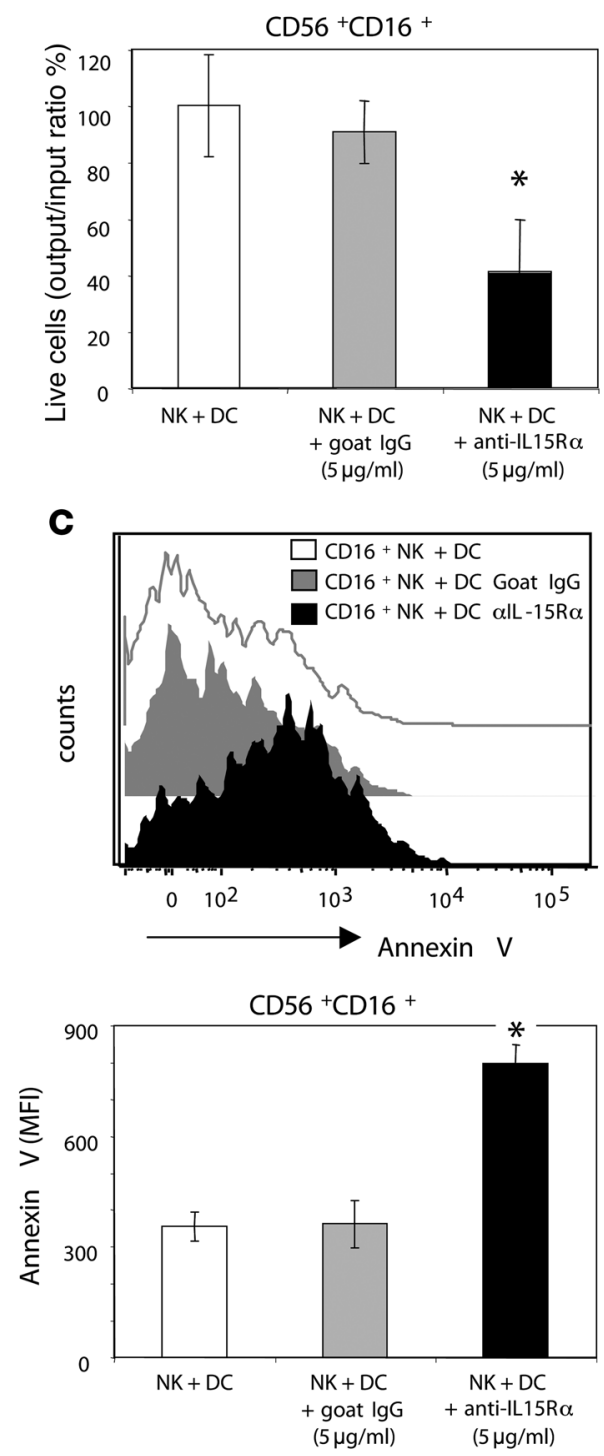

Figure 8

Blocking of IL-15R $\alpha$ decreased NK cell survival and upregulated annexin $\mathrm{V}$ binding on both NK cell subpopulations. (A and B) Blood NK cells were purified and treated for $6 \mathrm{~h}$ with polyclonal anti-IL-15R $\alpha$ or control goat IgG, and cocultured with poly(I:C)-matured DCs during 9 days, counted, stained, and analyzed by flow cytometry. Results are mean \pm SD of $(A)$ ratios of output to input of live NK cells normalized to controls or (B) absolute numbers of live cells compared with controls in at least 3 experiments ran in triplicate. ${ }^{*} P<0.001$ versus control (NK $+D C)$. NK cells were gated on size, $7 \mathrm{AAD}^{-}, \mathrm{CD}^{-}, \mathrm{CD}_{16+/-}^{+}$, and CD56+. (C) Annexin $\mathrm{V}$ binding was analyzed with and without blocking of IL-15R $\alpha$. Representative data from at least 3 different experiments are shown. (D) Annexin $V$ binding on the surface of both NK cell subsets was determined. Results are mean $\pm S D$ of mean fluorescence intensity (MFI) of annexin $\mathrm{V}$ staining. ${ }^{*} P<0.02$ versus control (NK + DC). Data are representative of 3 experiments. in DC/NK cell synapse organization. With respect to the function of this early IL-15R $\alpha$ polarization, 2 models are currently debated. In the first model, binding of IL-15 to IL-15R $\alpha$ - in the absence of the $\beta$ and $\gamma_{c}$ chains - does not transduce an activating signal to the IL-15-binding cell, but presents IL-15 to $\beta$ and $\gamma_{c}$ chain-carrying cells like $\mathrm{NK}$ cells and $\mathrm{CD}^{+} \mathrm{T}$ cells (trans-signaling). Indeed, IL-15R $\alpha$ on the surface of human monocytes has been shown to present IL-15 on the surface to $\mathrm{CD}^{+} \mathrm{T}$ cells (31). In animal studies, IL-15R $\alpha$ on bone marrow-derived cells supports survival of IL-15R $\alpha^{-}$NK cells in the periphery $(52,53)$. We also detected IL-15R $\alpha$ at the surface of mDCs, and the expression correlated with the maturation status of the cells (24) (T. Strowig, unpublished observations), but we did not observe IL-15R $\alpha$ polarization in $\mathrm{mDCs}$ within $\mathrm{DC} / \mathrm{NK}$ cell conjugates. This might have been the result of high IL-15R $\alpha$ expression levels in mDCs, exceeding the required IL-15R $\alpha$ amount necessary for NKIS formation. Such partial IL-15R $\alpha$ polarization to the DC/NK cell interface would not have been detected in our analysis. In the second model, IL-15R $\alpha$, as part of the trimeric IL-15R, assists in binding of IL-15 with high affinity (cis-signaling). In addition, recent work has suggested that IL-15 triggers additional signaling pathways that use IL-15R $\alpha$, but do not require the expression of $\beta$ and $\gamma_{c}$ chains $(54,55)$. These additional signaling pathways could be triggered in blood NK cells, because gene expression profiling has previously shown IL-15R $\alpha$ expression in both NK cells subpopulations (56), and in the present study we observed IL-15R $\alpha$ protein at the surface and within the cytoplasm of NK cells. From these 2 models and the observed IL-15R $\alpha$ expression on both DCs and NK cells, IL-15R $\alpha$ could be required on DCs (trans-signaling) and/or NK cells (cis-signaling) for NK cell survival upon DC-mediated stimulation. Furthermore, our IL-15R $\alpha$-specific Ab blocking, which increased NK cell apoptosis and decreased NK cell survival in the DC/NK cell cocultures, also did not distinguish between a requirement for IL-15R $\alpha$ on DCs versus NK cells. Because we were only able to partially silence the expression of the IL-15R $\alpha$ on mDCs using siRNAs techniques ( $40 \%-60 \%$ reduction), we could not address the requirement for IL-15R $\alpha$ on DCs for NK cell survival. However, the lack of IL-15R $\alpha$ on NK cells of individuals with a history of IM revealed the need for this molecule on NK cells to induce optimal survival after DC encounter. Of interest, in vitro- 

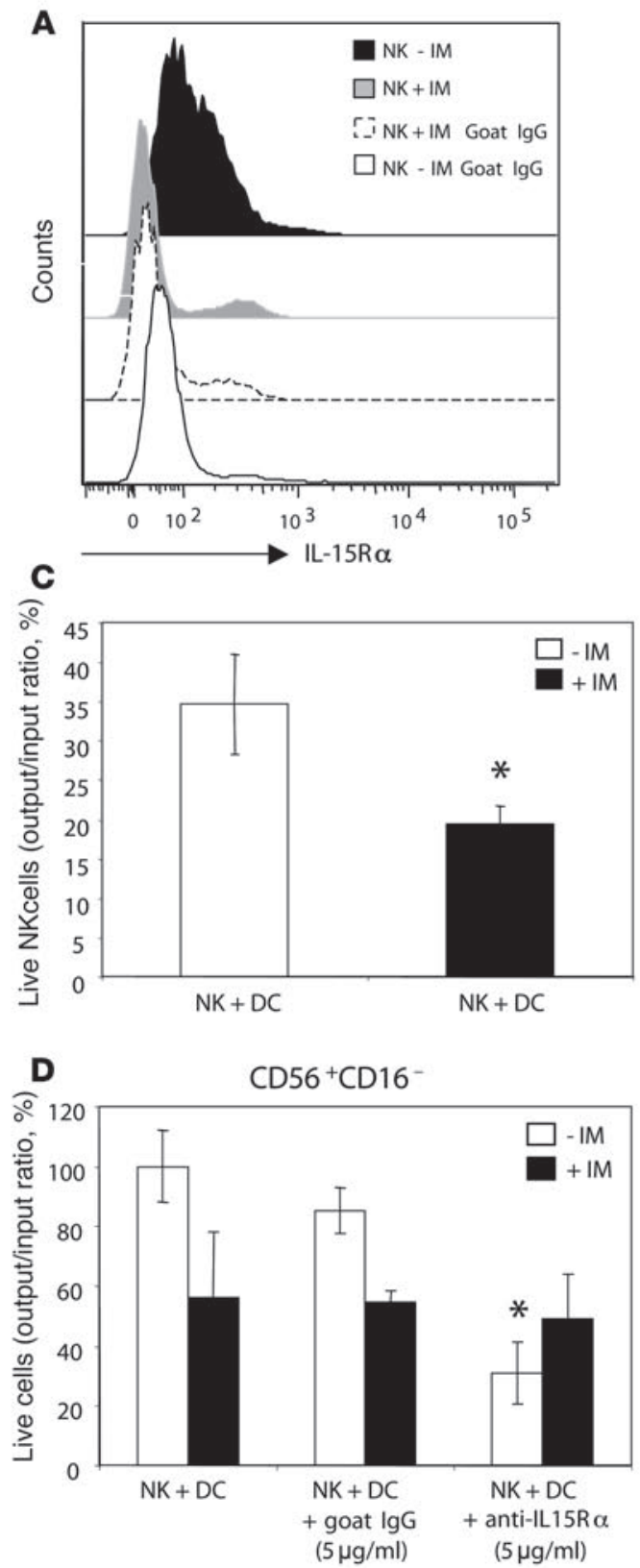

$\mathbf{B}$

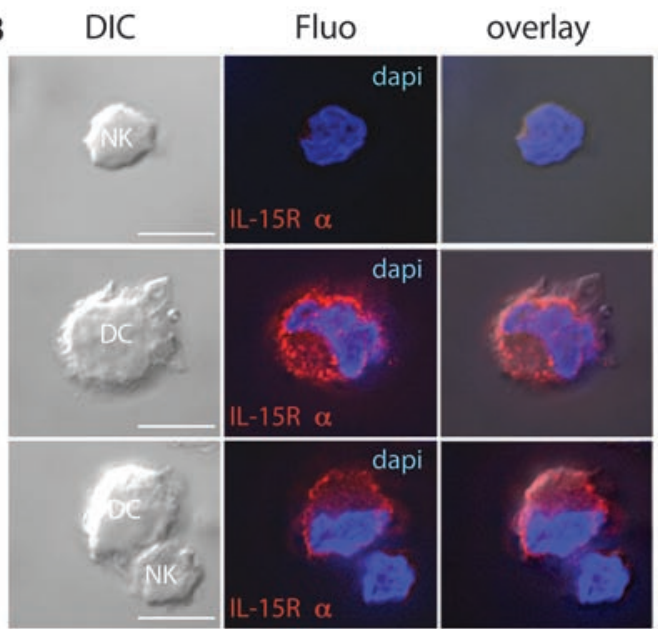

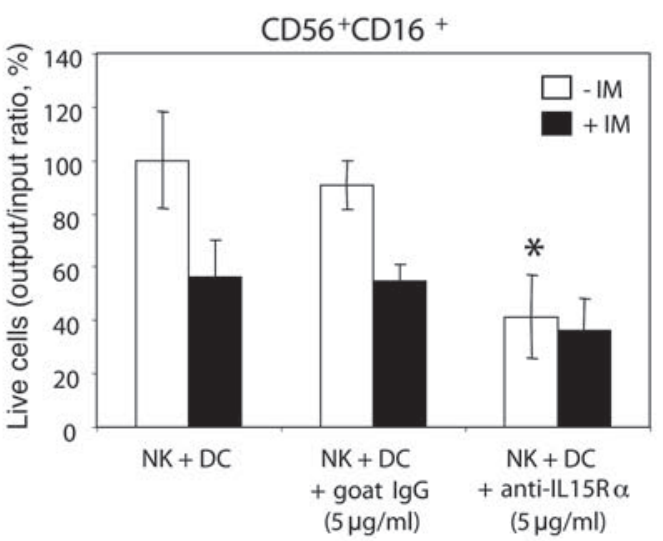

Figure 9

The expression of IL-15R $\alpha$ on NK cells correlated with their survival during DC/NK cell interactions. (A and B) NK cells from healthy donors with $(+)$ or without $(-)$ history of IM were isolated from blood and stained for IL-15R $\alpha$ before analysis by flow cytometry. (A) NK cells were gated on size, FSC/SSC singlets, CD3-, CD19-, and CD56+. Representative data from 2 separate experiments are shown. (B) Cells were also conjugated with poly(I:C)-matured autologous mDCs, and immunofluorescence images for presence and polarization of IL-15R $\alpha$ were obtained. Representative data from evaluation of 60 conjugates are shown. Scale bars: $5 \mu \mathrm{m}$. (C and D) Cocultures between NK cells and mDCs were performed for 9 days with and without blocking IL-15R $\alpha$. (C) Ratio of output to input of total NK cell numbers of donors with and without IM history were compared. (D) Ratios between output and input of live NK cells of healthy individuals with and without IM history after blocking of IL-15R $\alpha$. NK cells treated with normal goat IgG were used as control. NK cells were gated on size, 7AAD-, CD3 ${ }^{-}, C D_{16}^{+/-}$, and CD56 $6^{+}$. Data are mean \pm SD from 3 experiments in duplicate $\left(n=3-I M\right.$ donors; $2+I M$ donors). ${ }^{*} P<0.01$ versus control $(N K+D C)$.

differentiated Langerhans cells, producing higher levels of IL-15 compared with monocyte-derived DCs but lacking expression of IL-15R $\alpha$ on their surface, are still able to support survival of resting NK cells (46). These findings suggest that polarized IL-15R $\alpha$ plays an important role at the surface of NK cells during interactions with mDCs. IL-15R $\alpha$ on NK cells at the synapse with DCs could trigger additional signaling pathways in NK cells, which are essential for their survival, or capture IL-15 by the high affinity of the trimeric IL-15R complex from DCs that present IL-15 via IL-15R $\alpha$, for more efficient cis-signaling of this cytokine.

With respect to the relevance of NK cell survival induction by mature DCs via IL-15, distinct subsets of NK cells have been 
characterized in mice as mediators of innate immune control of murine CMV or hapten-induced contact hypersensitivity $\left(\mathrm{Ly}-49 \mathrm{H}^{+}\right.$ NK cells and Ly-49C-I+ NK cells, respectively; refs. 57-60). Longlasting expansion of these distinct NK cell subpopulations has been reported - at least for hapten-specific NK cells - to mediate protection from rechallenge for up to 4 weeks (60). This longlasting preparedness by maintenance of expanded NK cell subsets might be mediated by DCs that have been matured in response to viral infections. The prototypic viral maturation stimulus, doublestranded RNA, or its mimic, poly(I:C), might confer the ability to DCs to mediate survival of selectively expanded NK cells. Therefore, we propose that $\mathrm{mDCs}$ assist (via cell contact-dependent interactions with NK cells) in the maintenance of NK cell subsets that have expanded in response to specific antigenic challenge in order to ensure durable vigilance of the innate immune system against recently encountered pathogens.

In summary, we showed that poly(I:C)-matured DCs possessed a strong capacity to interact and activate resting NK cells, via the formation of what we believe to be a novel type of regulatory synapse between NK cells and DCs that displays features of both activating and inhibitory NK cell synapses. We propose that IL-15R $\alpha$, required on NK cells for optimal DC/NK cell interaction, mediates cell contact-dependent components of human NK cell survival induction by $\mathrm{mDC}$ to maintain NK cell vigilance against recently encountered pathogens.

\section{Methods}

$A b s$ and labeling reagents. Directly labeled anti-CD3 (clone SK7), antiCD11c (B-ly6), anti-CD56 (B-159), anti-CD69 (FN50), anti-CD86 (IT2.2), anti-CD94 (HP-3D9), anti-HLA-DR (TU36), annexin V, and 7AAD were obtained from BD Biosciences. Anti-CD16 (3G8) was purchased from Invitrogen. Anti-CD83 (HB15a), anti-LFA-1 (25.3.1), anti-CD158b1/b2,j (clone GL183, KIR2DL2, KIR2DL3, and KIR2DS2), anti-CD158a, h (clone EB6B, KIR2DL1, and KIR2DS1), and anti-NKG2A (Z199) were obtained from Beckman Coulter. Unconjugated Abs anti-IL-15R $\alpha$ (clone 151303) and goat polyclonal anti-IL-15R $\alpha$ were used from R\&D Systems. Anti-talin (TD77) and anti-perforin ( $(G 9)$ were purchased from Chemicon International. Rabbit and goat polyclonal anti-talin was purchased from Santa Cruz Biotechnology Inc. MHC class I stainings were performed with an anti-HLA-A, -B, and -C mAb (W6/32), derived from the supernatant of an ATCC hybridoma cell line. Blocking Abs for adhesion molecules used were anti-LFA-3 (TS2/9) and anti-ICAM-1 (W-CAM-1), both from Pierce, and rabbit polyclonal anti-ICAM-2 and anti-ICAM-3 (clone 76205), both from R\&D Systems. Cells labeled with unconjugated Abs were stained with Alexa Fluor 488-conjugated rabbit anti-goat IgG, Alexa Fluor 488-conjugated rabbit anti-mouse IgG, Alexa Fluor 555-conjugated goat anti-mouse IgG, Alexa Fluor 647-conjugated donkey anti-goat IgG (Invitrogen), or Rhodamine Red-X-conjugated donkey anti-goat or anti-rabbit (Jackson ImmunoResearch Laboratories). Actin staining was performed with the bodipy-conjugated pallacidin reagent from Invitrogen. For KIRs staining, an additional step of signal amplification was performed using biotinconjugated anti-mouse IgGs (Vector Laboratories) followed by staining with Alexa Fluor 555-conjugated Avidin (Invitrogen) on slides preblocked with an avidin/biotin blocking kit (Zymed Laboratories Inc.). Labeling of cells prior to conjugation assays was performed with Cell Trace Far Red DDAO-SE reagent or with INDO-1-AM (both from Invitrogen) prior to intracellular calcium measurements. Control Abs for stainings were normal goat IgG (R\&D Systems), mouse IgG1 (MOPC-21), mouse IgG2b, $\mathrm{k}$ (MOPC-141), and mouse IgG2a,K (UPC-10) from Sigma-Aldrich. Control Abs for cocultures were endotoxin- and azide-free normal goat IgG (R\&D
Systems), mouse IgG1 (MOPC-21), mouse IgG2b,к (MPC-11), and mouse IgG2a,K (MOPC-173) from BioLegend.

Human monocyte-derived DCs and NK cells. PBMCs were isolated from leukocyte concentrates (New York Blood Center) and blood from healthy donors with or without history of IM by density-gradient centrifugation on Ficoll/Hypaque. CD14 ${ }^{+}$cells were isolated from PBMCs by positive magnetic cell separation (MACS; Miltenyi Biotec) and cultured for 5 days in RPMI1640, 1\% heat-inactivated human AB serum (Cellgro), IL-4, and GM-CSF, according to standard protocols (46). The CD14- cells were frozen for later isolation of NK cells. Monocyte-derived DCs were matured for 2 days in medium with IL-4, GM-CSF, and $25 \mu \mathrm{g} / \mathrm{ml}$ poly(I:C) (Invivogen). Maturation of DCs was monitored by flow cytometry using anti-CD11c, anti-CD83, anti-CD86, and anti-HLA-DR Abs.

Frozen CD14- PBMCs were thawed and washed, and NK cells were isolated from $\mathrm{CD} 14^{-}$fractions by negative selection using the NK cell Isolation Kit II (MACS; Miltenyi Biotec) according to the manufacturer's instructions. The purity of the isolated NK cells was higher than $90 \%$ and contained less than $5 \%$ contaminating $\mathrm{T}$ cells as determined by flow cytometry. Where indicated, NK cells were then stained with anti-CD16, anti-CD56, and anti-CD3 and further purified into the 2 main NK cell subpopulations, CD56 ${ }^{\text {bright }} \mathrm{CD} 16^{-}$and $\mathrm{CD} 56^{\mathrm{dim}} \mathrm{CD} 16^{+}$, by flow cytometric sorting (FACSVantage; BD Biosciences). The number of sorted cells was adjusted prior to conjugation assays.

Human DC/NK cell cocultures and conjugation assays. Directly after isolation, resting NK cells were cultured with autologous DCs at a ratio of 5:1 for $2 \mathrm{~h}$ to $9 \mathrm{~d}$ at $37^{\circ} \mathrm{C}$ in RPMI1640, $1 \%$ human $\mathrm{AB}$ serum, and gentamicin. In IL-15R $\alpha$-blocking experiments, resting NK cells were cultured for $6 \mathrm{~h}$ alone with isotype control $\mathrm{Ab}(5 \mu \mathrm{g} / \mathrm{ml})$ or blocking $\mathrm{Abs}(5 \mu \mathrm{g} / \mathrm{ml})$ before coculturing with DCs. In synapse-blocking experiments, $\mathrm{mDCs}$ were pretreated during $2 \mathrm{~h}$ with isotypes and blocking $\mathrm{Abs}(5 \mu \mathrm{g} / \mathrm{ml})$ before adding NK cells. Abs were then added again on day 4 of culture in both kinds of experiments. Where indicated, DCs (bottom) were separated from NK cells (top) by $0.4-\mu \mathrm{m}$ pore membranes (Corning). At the end of the culture, the number of live cells was determined by trypan blue exclusion, and cells were then stained for flow cytometry with Abs and 7AAD. Live cell numbers at day 9 were recalculated according to frequencies of NK cells after flow analysis. Numbers of live cells at the end of the cultures (output) were compared with number of cells added initially (input). Values were then transformed into percentages relative to the control condition $(\mathrm{NK}+\mathrm{DC})$. For conjugation assays, resting NK cells were conjugated to imDCs or poly(I:C)-matured DCs. Cells were mixed at a NK cell/DC ratio of $2: 1$ in $20 \mu \mathrm{l}$ of RPMI1640 without serum, prior to being pelleted quickly at $10,000 \mathrm{~g}$. The pellets were then allowed to conjugate for the indicated time periods at $37^{\circ} \mathrm{C}$. After conjugation, cells were gently resuspended in RPMI1640 prior to intracellular calcium measurements by flow cytometry analysis or centrifugated on poly-lysine-coated 1.5 -mm coverslips for immunofluorescence analyses.

Where indicated, purified NK cells were activated overnight with IL-2 (500 IU $/ 10^{6}$ cells, Proleukine; Chiron) prior to conjugation with Cell Trace Far Red DDAO-SE-labeled target cell LCL721.221 (MHC class I-negative EBV-B lymphoblastoid cell line).

Immunofluorescence microscopy and analysis. Cells on slides were fixed in 3\% paraformaldehyde for $20 \mathrm{~min}$ at $4^{\circ} \mathrm{C}$. Cells were permeabilized with $0.01 \%$ Triton-X for $1 \mathrm{~min}$ at room temperature. Cells were then stained with the indicated Abs in the presence of 5\% species-appropriate serum, followed by the appropriate secondary reagent. All washes were performed in PBS supplemented with $1 \%$ fish skin gelatin (Sigma-Aldrich) and $0.02 \%$ saponin (Sigma-Aldrich). After staining, slides were counterstained with DAPI and mounted with Prolong gold anti-fade reagent (Invitrogen). Slides were visualized through a $\times 1001.4$ NA oil immersion lens with an inverted Olympus 
IX-70 microscope (DeltaVision Image Restoration Microscope; Applied Precision/Olympus) and a Photometrics CoolSnap QE camera. Serial optical sections ( $0.2 \mu \mathrm{m} ; 60-70$ sections) were acquired for all labelings. Images were then deconvolved using DeltaVision SoftWoRx software version 3.4.4., and pictures were overlaid using Metamorph software version 6.3r6 (Universal Imaging Corporation). Three-dimensional analyses were performed after deconvolution, and chromatical aberration was corrected using SoftWoRx software. Images were exported as .TIF files. Percent values were determined by evaluation of 100-200 conjugates in randomly selected fields in at least 3 separate experiments. NK cells in DC/NK cell or LCL721.221/NK cell conjugates were identified using: (a) differential interference contrast (DIC; also known as Nomarski) images, in which differences in cell size were obvious; (b) chromatin density, in which DAPI staining stains brightly condensed and round-shaped nuclei of NK cells as opposed to less dense DC nuclei; (c) cytoplasm area, in which DCs have a large area of cytoplasm and the ratio between cytoplasm and nucleus is small in NK cells; and (d) Cell Trace Far Red DDAO-SE labeling of NK cells or LCL721.221 cells. For 3D synapse analysis, stacks were rebuilt, and orthogonal views of the Z-stack were visualized with Metamorph. Analyses evaluating the distribution of voxels within the 3D-reconstructed synapse (as shown in Figure 6D) were realized using the mask function of ImageJ software, version $1.38 \mathrm{n}(\mathrm{NIH})$. Percentages of fluorescence colocalized per unit of volume were calculated after setting a threshold of $31 \%$ of the dynamic range for the red channel (IL-15R $\alpha$ or KIRs) and $29 \%$ of the dynamic range for the green channel (CD94 or IL-15R $\alpha$ ). Notably, the area of the region used to highlight the peripheral synapse (Figure 6C, top 4 rows) was 4 -fold greater than the one for the central synapse (Figure 6C, two bottom rows). Figure 5E was obtained using a $\times 1001.4$ NA oil immersion lens with a Zeiss LSM 510 confocal microscope (Carl Zeiss MicroImaging Inc.), and images were acquired and analyzed using LSM 510 software version 3.2 SP2.

Quantification of molecules at the synapse in conjugates was realized after image analysis with ImageJ software. We measured the enrichment of a molecule at the contact site of each conjugate compared with the distribution of the same molecule in a similar area opposite to the contact site within the same cell. The relative enrichment (RE) was calculated as the average intensity per unit volume at the contact site or at the opposite membrane across the contact site divided by the average intensity per unit volume of the entire cell. The area of the contact site was defined manually and represented $16.2 \% \pm 5.45 \%$ of the total cell volume. The area across the contact site was defined manually and represented $16.5 \% \pm 5.58 \%$ of the total cell volume. The values obtained in unconjugated cells were considered as baseline, and data represent the ratio of RE at the contact site to RE at the membrane across the contact site normalized to the values in single cells, assigned as $100 \%$.

1. Moretta, L., et al. 2002. Human natural killer cells: their origin, receptors and function. Eur. J. Immunol. 32:1205-1211.

2. Moretta, A., et al. 2001. Activating receptors and coreceptors involved in human natural killer cell-mediated cytolysis. Annu. Rev. Immunol. 19:197-223.

3. Natarajan, K., et al. 2002. Structure and function of natural killer cell receptors: multiple molecular solutions to self, nonself discrimination. Annu. Rev. Immunol. 20:853-885

4. Borrego, F., Masilamani, M., Kabat, J., Sanni, T.B., and Coligan, J.E. 2005. The cell biology of the human natural killer cell CD94/NKG2A inhibitory receptor. Mol. Immunol. 42:485-488.

5. Vyas, Y.M., Maniar, H., and Dupont, B. 2002. Visualization of signaling pathways and cortical cytoskeleton in cytolytic and noncytolytic natural killer cell immune synapses. Immunol. Rev. 189:161-178.

6. Grakoui, A., et al. 1999. The immunological synapse: a molecular machine controlling $T$ cell activa-

Flow cytometry and intracellular calcium measurements. After coculture, cells were washed and stained with the indicated combinations of Abs and then analyzed by flow cytometry. For intracellular calcium measurements, freshly isolated resting NK cells were loaded with $5 \mu \mathrm{M}$ INDO-1$\mathrm{AM}$ (Invitrogen) for $30 \mathrm{~min}$ at $37^{\circ} \mathrm{C}$ in the dark, then washed twice with excess RPM1 1640 and stained with anti-CD3 and anti-CD56. Basal levels of $\left[\mathrm{Ca}^{2+}\right]$ in unconjugated INDO-1-AM-loaded NK cells were recorded during $30 \mathrm{~s}$, after which acquisition was paused and INDO-1-loaded NK cells were retrieved and mixed with mDCs. Cells were incubated for 1 or $6 \mathrm{~min}$ at $37^{\circ} \mathrm{C}$, then flow acquisition was appended, and the ratio between free and bound $\mathrm{Ca}^{2+}$ was determined by measuring changes in the fluorescence of NK cells. Data show the mean value of the response and are representative data from 3 experiments. Cells were acquired on a BD LSR II cytometer using FACSDIva software (BD Biosciences) and all flow cytometry analyses were analyzed with FlowJo software version 6.4.4 (Tristar). Notably, the export of data from FACSDIva software to FlowJo software results in a compression of the lower intensities of mean fluorescence between 0 and 100 with no omission of information (see Figure 1 for an example).

Statistics. Statistical analyses were performed with the paired 2-tailed Student's $t$ test. A $P$ value less than 0.05 was considered significant. Plotted data are displayed as mean and SD unless otherwise indicated.

\section{Acknowledgments}

The authors thank Y. Vyas, W. Huang, D. Schmid, B. Morandi, I. Mecklenbrauker, J. Lünemann, and C. Paludan for advice and protocols; K. Velinzon for flow cytometry sorting; and K. Heller for recruitment of blood donors. We are especially grateful to A. North, D. Elreda, and M. Marchand for helpful discussion and advice about image analyses. This work was supported by the Arnold and Mabel Beckman Foundation, the Alexandrine and Alexander Sinsheimer Foundation, the Burroughs Wellcome Fund, and the National Cancer Institute (R01CA108609 and R01CA101741), as well as in part by an Institutional Clinical and Translational Science Award (CTSA) to the Rockefeller University Hospital (U54RR023419).

Received for publication February 8, 2007, and accepted in revised form August 15, 2007.

Address correspondence to: Christian Münz, Laboratory of Viral Immunobiology, Rockefeller University, 1230 York Avenue, New York, New York 10065, USA. Phone: (212) 327-7611; Fax: (212) 327-7887; E-mail:munzc@rockefeller.edu.

tion. Science. 285:221-227.

7. Vyas, Y.M., Maniar, H., and Dupont, B. 2002. Cutting edge: differential segregation of the SRC homology 2-containing protein tyrosine phosphatase-1 within the early NK cell immune synapse distinguishes noncytolytic from cytolytic interactions. J. Immunol. 168:3150-3154.

8. Davis, D.M., et al. 1999. The human natural killer cell immune synapse. Proc. Natl. Acad. Sci. U. S. A. 96:15062-15067.

9. Vyas, Y.M., et al. 2001. Spatial organization of signal transduction molecules in the NK cell immune synapses during MHC class I-regulated noncytolytic and cytolytic interactions. J. Immunol. 167:4358-4367.

10. Krzewski, K., Chen, X., Orange, J.S., and Strominger, J.L. 2006. Formation of a WIP-, WASp-, actin-, and myosin IIA-containing multiprotein complex in activated NK cells and its alteration by KIR inhibitory signaling. J. Cell Biol. 173:121-132.

11. Sanni, T.B., Masilamani, M., Kabat, J., Coligan, J.E., and Borrego, F. 2004. Exclusion of lipid rafts and decreased mobility of CD94/NKG2A receptors at the inhibitory NK cell synapse. Mol. Biol. Cell. 15:3210-3223.

12. McCann, F.E., et al. 2003. The size of the synaptic cleft and distinct distributions of filamentous actin, ezrin, CD43, and CD45 at activating and inhibitory human NK cell immune synapses. J. Immunol. 170:2862-2870.

13. Cooper, M.A., Fehniger, T.A., and Caligiuri, M.A. 2001. The biology of human natural killer-cell subsets. Trends Immunol. 22:633-640.

14. Cooper, M.A., et al. 2001. Human natural killer cells: a unique innate immunoregulatory role for the CD56 bright subset. Blood. 97:3146-3151.

15. Jacobs, R., et al. 2001. CD56 $6^{\text {bright }}$ cells differ in their KIR repertoire and cytotoxic features from CD56 dim NK cells. Eur. J. Immunol. 31:3121-3127.

16. Ferlazzo, G., and Münz, C. 2004. NK cell compartments and their activation by dendritic cells. 
J. Immunol. 172:1333-1339.

17. Vitale, M., et al. 2004. The small subset of CD56 ${ }^{\text {bright }} \mathrm{CD} 16^{-}$natural killer cells is selectively responsible for both cell proliferation and interferon-gamma production upon interaction with dendritic cells. Eur. J. Immunol. 34:1715-1722.

18. Andrews, D.M., Scalzo, A.A., Yokoyama, W.M., Smyth, M.J., and Degli-Esposti, M.A. 2003. Functional interactions between dendritic cells and NK cells during viral infection. Nat. Immunol 4:175-181.

19. Kassim, S.H., Rajasagi, N.K., Zhao, X., Chervenak, R., and Jennings, S.R. 2006. In vivo ablation of CD11c-positive dendritic cells increases susceptibility to herpes simplex virus type 1 infection and diminishes NK and T-cell responses. J. Virol. 80:3985-3993.

20. Lucas, M., Schachterle, W., Oberle, K., Aichele, P., and Diefenbach, A. 2007. Dendritic cells prime natural killer cells by trans-presenting interleukin 15. Immunity. 26:503-517.

21. Degli-Esposti, M.A., and Smyth, M.J. 2005. Close encounters of different kinds: dendritic cells and NK cells take centre stage. Nat. Rev. Immunol. 5:112-124.

22. Moretta, A., et al. 2005. Early liaisons between cells of the innate immune system in inflamed peripheral tissues. Trends Immunol. 26:668-675.

23. Bajenoff, M., et al. 2006. Natural killer cell behavior in lymph nodes revealed by static and real-time imaging. J. Exp. Med. 203:619-631.

24. Ferlazzo, G., et al. 2004. Distinct roles of IL-12 and IL-15 in human natural killer cell activation by dendritic cells from secondary lymphoid organs. Proc. Natl. Acad. Sci. U. S. A. 101:16606-16611.

25. Gerosa, F., et al. 2002. Reciprocal activating interaction between natural killer cells and dendritic cells. J. Exp. Med. 195:327-333.

26. Piccioli, D., Sbrana, S., Melandri, E., and Valiante, N.M. 2002. Contact-dependent stimulation and inhibition of dendritic cells by natural killer cells. J. Exp. Med. 195:335-341.

27. Koka, R., Burkett, P., Chien, M., Chai, S., Boone, D.L., and Ma, A. 2004. Cutting edge: murine dendritic cells require IL-15R alpha to prime NK cells. J. Immunol. 173:3594-3598.

28. Lodolce, J.P., et al. 1998. IL-15 receptor maintains lymphoid homeostasis by supporting lymphocyte homing and proliferation. Immunity. 9:669-676.

29. Kennedy, M.K., et al. 2000. Reversible defects in natural killer and memory CD8 T cell lineages in interleukin 15-deficient mice. J. Exp. Med. 191:771-780.

30. Giron-Michel, J., et al. 2005. Membrane-bound and soluble IL-15/IL-15Ralpha complexes display differential signaling and functions on human hematopoietic progenitors. Blood. 106:2302-2310.

31. Dubois, S., Mariner, J., Waldmann, T.A., and Tagaya, Y. 2002. IL-15Ralpha recycles and presents IL-15 In trans to neighboring cells. Immunity. 17:537-547.

32. Vamosi, G., et al. 2004. IL-2 and IL-15 receptor alpha-subunits are coexpressed in a supramolecular receptor cluster in lipid rafts of T cells. Proc. Natl. Acad. Sci. U. S. A. 101:11082-11087.

33. Borg, C., et al. 2004. NK cell activation by dendritic cells (DCs) requires the formation of a synapse leading to IL-12 polarization in DCs. Blood. 104:3267-3275.

34. Semino, C., Angelini, G., Poggi, A., and Rubartelli, A. 2005. NK/iDC interaction results in IL-18 secretion by DCs at the synaptic cleft followed by NK cell activation and release of the DC maturation factor HMGB1. Blood. 106:609-616.

35. Anikeeva, N., et al. 2005. Distinct role of lymphocyte function-associated antigen-1 in mediating effective cytolytic activity by cytotoxic $\mathrm{T}$ lymphocytes. Proc. Natl. Acad. Sci. U. S. A. 102:6437-6442.

36. Lanier, L.L. 1998. NK cell receptors. Annu. Rev. Immunol. 16:359-393.

37. Borrego, F., et al. 2002. Structure and function of major histocompatibility complex (MHC) class I specific receptors expressed on human natural killer (NK) cells. Mol. Immunol. 38:637-660.

38. Ferlazzo, G., et al. 2004. The abundant NK cells in human secondary lymphoid tissues require activation to express killer cell Ig-like receptors and become cytolytic. J. Immunol. 172:1455-1462.

39. Bamford, R.N., et al. 1994. The interleukin (IL) 2 receptor beta chain is shared by IL-2 and a cytokine, provisionally designated IL-T, that stimulates T-cell proliferation and the induction of lymphokine-activated killer cells. Proc. Natl. Acad. Sci. U. S. A. 91:4940-4944.

40. Burton, J.D., et al. 1994. A lymphokine, provisionally designated interleukin $\mathrm{T}$ and produced by a human adult T-cell leukemia line, stimulates T-cell proliferation and the induction of lymphokineactivated killer cells. Proc. Natl. Acad. Sci. U. S. A. 91:4935-4939.

41. Waldmann, T.A., and Tagaya, Y. 1999. The multifaceted regulation of interleukin- 15 expression and the role of this cytokine in NK cell differentiation and host response to intracellular pathogens. Annu. Rev. Immunol. 17:19-49.

42. Sauce, D., et al. 2006. EBV-associated mononucleosis leads to long-term global deficit in T-cell responsiveness to IL-15. Blood. 108:11-18.

43. Moretta, A. 2002. Natural killer cells and dendritic cells: rendezvous in abused tissues. Nat. Rev. Immunol. 2:957-964.

44. Leon, B., Lopez-Bravo, M., and Ardavin, C. 2007. Monocyte-derived dendritic cells formed at the infection site control the induction of protective $\mathrm{T}$ helper 1 responses against leishmania. Immunity. 26:519-531.

45. Gerosa, F., et al. 2005. The reciprocal interaction of NK cells with plasmacytoid or myeloid dendritic cells profoundly affects innate resistance functions. J. Immunol. 174:727-734.

46. Münz, C., et al. 2005. Mature myeloid dendritic cell subsets have distinct roles for activation and viability of circulating human natural killer cells. Blood. 105:266-273.

47. Serbina, N.V., Salazar-Mather, T.P., Biron, C.A., Kuziel, W.A., and Pamer, E.G. 2003. TNF/iNOSproducing dendritic cells mediate innate immune defense against bacterial infection. Immunity. 19:59-70

48. Fernandez, N.C., et al. 1999. Dendritic cells directly trigger NK cell functions: cross-talk relevant in innate anti-tumor immune responses in vivo. Nat. Med. 5:405-411.

49. Orange, J.S., et al. 2003. The mature activating natural killer cell immunologic synapse is formed in distinct stages. Proc. Natl. Acad. Sci. U. S. A. 100:14151-14156.

50. Ferlazzo, G., et al. 2002. Human dendritic cells activate resting natural killer (NK) cells and are recognized via the NKp30 receptor by activated NK cells. J. Exp. Med. 195:343-351.

51. Della Chiesa, M., et al. 2003. The natural killer cellmediated killing of autologous dendritic cells is confined to a cell subset expressing CD94/NKG2A, but lacking inhibitory killer Ig-like receptors. Eur. J. Immunol. 33:1657-1666.

52. Burkett, P.R., et al. 2004. Coordinate expression and trans presentation of interleukin (IL)-15Ralpha and IL-15 supports natural killer cell and memory $\mathrm{CD}^{+} \mathrm{T}$ cell homeostasis. J. Exp. Med. 200:825-834.

53. Koka, R., et al. 2003. Interleukin (IL)-15Ralphadeficient natural killer cells survive in normal but not IL-15Ralpha-deficient mice. J. Exp. Med. 197:977-984.

54. Dubois, S., et al. 2003. Distinct pathways involving the FK506-binding proteins 12 and 12.6 underlie IL-2-versus IL-15-mediated proliferation of T cells. Proc. Natl. Acad. Sci. U. S. A. 100:14169-14174.

55. Bulanova, E., et al. 2001. The IL-15R alpha chain signals through association with Syk in human B cells. J. Immunol. 167:6292-6302.

56. Hanna, J., et al. 2004. Novel insights on human NK cells' immunological modalities revealed by gene expression profiling. J. Immunol. 173:6547-6563.

57. Arase, H., Mocarski, E.S., Campbell, A.E., Hill, A.B., and Lanier, L.L. 2002. Direct recognition of cytomegalovirus by activating and inhibitory NK cell receptors. Science. 296:1323-1326.

58. Daniels, K.A., et al. 2001. Murine cytomegalovirus is regulated by a discrete subset of natural killer cells reactive with monoclonal antibody to Ly $49 \mathrm{H}$. J. Exp. Med. 194:29-44.

59. Lee, S.H., et al. 2001. Susceptibility to mouse cytomegalovirus is associated with deletion of an activating natural killer cell receptor of the C-type lectin superfamily. Nat. Genet. 28:42-45.

60. O'Leary, J.G., Goodarzi, M., Drayton, D.L., and von Andrian, U.H. 2006. T cell- and B cell-independent adaptive immunity mediated by natural killer cells. Nat. Immunol. 7:507-516. 